\title{
Isolation and characterisation of silver(I) complexes of substituted coumarin-4-carboxylates which are effective against Pseudomonas aeruginosa biofilms
}

\author{
Maeve Sullivan $^{\mathrm{a}, \mathrm{b}}$, Agnieszka Folytn-Arfa Kia ${ }^{\mathrm{a}, \mathrm{b}}$, Mark Long ${ }^{\mathrm{a}, \mathrm{b}}$, Maureen Walsh ${ }^{\mathrm{a}, \mathrm{b}}$, Kevin Kavanagh ${ }^{\mathrm{c}}$, \\ Siobhán McClean ${ }^{\mathrm{a}, \mathrm{d}}$, Bernadette S. Creaven ${ }^{\mathrm{a}, \mathrm{b}, *}$ \\ ${ }^{a}$ Department of Science, Institute of Technology Tallaght, Dublin 24, Ireland \\ ${ }^{\mathrm{b}}$ Centre of Applied Science for Health, Institute of Technology Tallaght, Dublin 24, Ireland \\ ${ }^{\mathrm{c}}$ Biology Department, National University of Ireland, Maynooth, Co. Kildare, Ireland \\ ${ }^{\mathrm{d}}$ Centre for Microbial Host Interactions, Institute of Technology Tallaght, Dublin 24, Ireland
}

\section{A R T I C L E I N F O}

\section{Article history:}

Received 26 August 2013

Accepted 26 September 2013

Available online 15 October 2013

\section{Keywords:}

Pseudomonas aeruginosa

Biofilms

Silver complexes

Coumarin

\begin{abstract}
A B S T R A C T
A novel series of coumarin-4-carboxylate ligands (2a-2l), their $\mathrm{Ag}(\mathrm{I})$ complexes (3a-3i) together with a number of their phenanthroline adducts $(\mathbf{4 d}, \mathbf{4} \mathbf{f}$ and $\mathbf{4 g}$ ) have been synthesised and characterised. The ligands were prepared by either acid or base hydrolysis of their corresponding esters or by demethylation of a methylated acid derivative. The esters (1a-1i) were synthesised by an aromatic electrophilic substitution reaction between the conjugate base of substituted phenols and a vinyltriphenylphosphonium salt. The novel series of $\mathrm{Ag}(\mathrm{I})$ carboxylate complexes were prepared by reaction of silver nitrate with the coumarin ligands in aqueous/alcohol solution. The $\mathrm{Ag}(\mathrm{I})$ phenanthroline adducts were prepared by reaction of the $\mathrm{Ag}(\mathrm{I})$ complex with 1,10-phenanthroline in aqueous/alcohol solution. A selection of complexes were screened for their in vitro antibacterial activity against Pseudomonas aeruginosa grown planktonically or as a biofilm as well as a number of other clinically important strains, Escherichia coli, MRSA and Staphylococcus aureus. The activity against the P. aeruginosa biofilm was found to be significantly greater than against the planktonic bacteria.
\end{abstract}

(c) 2013 Elsevier Ltd. All rights reserved.

\section{Introduction}

The Centres for Disease Control and Prevention in America have estimated that 2 million patients suffer from hospital-acquired infections (HAIs) every year and from that number there are approximately 100,000 deaths [1]. Hospital acquired infections result in significant levels of illness, death and financial cost to global healthcare systems. Many HAIs are a side effect of medical treatments such as surgery and the use of implanted medical devices including prosthetic implants and central venous and urinary catheters [2]. For the most part of the last decade, Methicillin-resistant Staphylococcus aureus (MRSA) was the leading cause of HAIs in Ireland [3]. However, another bacterium, Pseudomonas aeruginosa, is of increasing concern worldwide with for example 200,000 HAIs directly related to that organism annually in the USA alone [4]. Bacteria such P. aeruginosa, Staphylococci and Escherichia coli that can exist in biofilms exhibit extreme resistance compared to planktonic or free moving bacteria as they are protected from the host's

\footnotetext{
* Corresponding author at: Department of Science, Institute of Technology Tallaght, Dublin 24, Ireland. Tel.: +353 (0)1 4042889.
}

E-mail address: Bernie.Creaven@ittdublin.ie (B.S. Creaven). immune cells and from the action of antimicrobial agents. It is reported that to kill bacteria in a biofilm requires 1000 times the antibiotic dose necessary to kill a planktonic population of cells [1]. Up to $65-80 \%$ of human infections are caused by biofilms and these are often the underlying reason why treatment with antibiotics can fail [5]. The ever-increasing problem of microbial resistance means that there is a need to develop effective strategies to prevent and control HAIs. One such approach is to synthesise new antimicrobial agents that effectively inhibit the growth of microorganisms and which can be used as additives in efficient surface technologies such as coatings and paints and in topical healthcare products. The use of antimicrobial coatings in the clinical environment can aid in reducing the risk of cross contamination and transmission of microbes and also help overcome antimicrobial drug resistance.

There is a renewed interest in the use of silver metal and silver(I) salts and compounds as antimicrobial agents. The antimicrobial activity of silver has long been known but the introduction of penicillin in the 1940s meant that antibiotics became the standard treatment for infections and silver was no longer used. In the 1960s silver nitrate was used to treat burn victims which led to the production of silver sulfadiazine cream [6,7]. Silver in its ionic form, 
$\mathrm{Ag}(\mathrm{I})$, is active against a broad range of microbes even at low concentrations. Silver displays broad spectrum activity in which it attacks multiple sites [8,9]. Studies have shown that the most important mechanism of action of $\mathrm{Ag}(\mathrm{I})$ is based on its surface binding and damage to the membrane function [10]. More recently there are a number of products (topical creams, wound dressings, sutures, catheters etc.) on the market that incorporate a form of silver to produce an antimicrobial effect. Topical treatments in the form of hydrogels such as Silvasorb [11] and Silver Shield [12] and wound dressings such as those from Acticoat ${ }^{\mathrm{TM}}$ [13], Aquacel ${ }^{\circledR}$ (acticoat) and Silvercel ${ }^{\circledR}$ are alternatives to antibiotic treatment. A number of silver antimicrobial additives manufactured for use in many applications include AgIon from AgIon [14], and Biomaster from Addmaster [15].

Whilst there are a number of biocidal silver products currently available on the market, there is still a need to develop more efficient cost effective silver-based products which offer long-term antimicrobial activity. Using $\mathrm{Ag}(\mathrm{I})$ complexes which contain both an organic (ligand) and inorganic (metal) component as the biocidal additive represents a novel approach. The use of $\mathrm{Ag}(\mathrm{I}) \mathrm{com}-$ plexes as biocidal additives in hygienic coatings has been demonstrated by our previous work in which $\mathrm{Ag}(\mathrm{I})$ complexes of coumarin-3-carboxylates were successfully incorporated into sol-gel derived coatings. The thin-film coatings were found to be effective against MRSA and Enterobacter cloacae WT6 strains at low silver loadings $(0.3 \% \mathrm{w} / \mathrm{w})$ and were less toxic than coatings containing silver nitrate and showed potential for use in the clinical environment [16].

In this present work we have prepared a series of novel 4-coumarin carboxylate $\mathrm{Ag}(\mathrm{I})$ complexes. Coumarin and its derivatives have shown a diverse array of biological activities including antimicrobial [17], chemotherapeutic [18,19], anticoagulant [20] and antiinflammatory [21]. A number of both naturally occurring and synthetic coumarins including aminocoumarins [22,23], hydroxycoumarins [24,25], pyranocoumarins [26], and furanocoumarins [27] have been reported to have significant antimicrobial activity against a wide range of microbes. Following on from our previous work on a series of $\mathrm{Ag}(\mathrm{I})$ complexes of coumarin-3-carboxylates, which have proven antimicrobial activity and potential as effective biocides in hygienic coatings, a novel series of $\mathrm{Ag}(\mathrm{I})$ complexes of coumarin-4-carboxylates has been synthesised and screened for their antibacterial activity. In addition, three $\mathrm{Ag}(\mathrm{I})$ complexes containing coumarin carboxylates and phenanthroline (phen) ligands were also isolated and their antimicrobial activity investigated.

\section{Experimental}

\subsection{Materials/general methods}

All chemicals purchased from Sigma-Aldrich were reagent grade and used without purification. Infrared spectra were recorded in the region of $4000-400 \mathrm{~cm}^{-1}$, on a Nicolet Impact 410 Fourier-Transform Infrared spectrophotometer using Omnic software. NMR spectra were obtained from both JOEL and Bruker spectrometers in $\mathrm{d}_{6}$-DMSO and were recorded in the region of -5 to $15 \mathrm{ppm}$ from TMS for ${ }^{1} \mathrm{H}$ NMR spectra and in the region of -33 to $233 \mathrm{ppm}$ for ${ }^{13} \mathrm{C}$ NMR spectra. NMR spectra run on a JOEL JNM-LA300 (300 MHz ${ }^{1} \mathrm{H}$ and $75 \mathrm{MHz}{ }^{13} \mathrm{C}$ ) spectrometer were recorded with a resolution of 0.0006 and $0.008 \mathrm{ppm}$ for ${ }^{1} \mathrm{H}$ NMR and ${ }^{13} \mathrm{C}$ NMR spectra respectively. NMR spectra run on a Bruker Avance III spectrometer $\left(300 \mathrm{MHz}{ }^{1} \mathrm{H}\right.$ and $75 \mathrm{MHz}{ }^{13} \mathrm{C}$ ) were recorded with a resolution of 0.000315 and $0.0037 \mathrm{ppm}$ for ${ }^{1} \mathrm{H}$ NMR and ${ }^{13} \mathrm{C}$ NMR respectively. NMR spectra run on a Bruker Avance III 500 spectrometer $\left(500 \mathrm{MHz}{ }^{1} \mathrm{H}\right.$ and $\left.125 \mathrm{MHz}{ }^{13} \mathrm{C}\right)$ were recorded with a resolution of 0.00022 and $0.0036 \mathrm{ppm}$ for ${ }^{1} \mathrm{H}$ NMR and ${ }^{13} \mathrm{C}$ NMR respectively. UV-Visible (UV-Vis) spectra of the compounds in DMSO were recorded on a Hitachi U-2001 Spectrophotometer. Atomic absorption spectroscopy (AAS) measurements were taken on a Perkin-Elmer 460 AAS instrument (emission wavelength $328.1 \mathrm{~nm}$ ). Microanalytical data were provided by the Microanalytical Laboratory, National University of Ireland Dublin, Belfield, Dublin 4. Melting point values were recorded using a Bibby SMP10 melting point apparatus and are uncorrected. Values were taken up to $300^{\circ} \mathrm{C}$. Molar conductivity was measured on a Systronic conductivity bridge with a diptype cell, using $4 \times 10^{-3} \mathrm{M}$ solution of compounds in DMSO.

\subsection{Syntheses of ligands}

\subsubsection{Synthesis of substituted esters $\mathbf{1 a - 1 i}$}

All of the esters 1a-1i (Fig. S1 in Supplementary data and Table 1) were synthesised using the following general procedure. The appropriate phenol $(40 \mathrm{mmol})$ and triphenylphosphine $(7.8 \mathrm{~g}$, $30 \mathrm{mmol}$ ) were dissolved with stirring in dichloromethane $(80 \mathrm{ml})$. To this a solution of dimethyl acetylenedicarboxylate $(5 \mathrm{ml}, 5.68 \mathrm{~g}, 40 \mathrm{mmol})$ in dichloromethane $(20 \mathrm{ml})$ was added dropwise over $10 \mathrm{~min}$ at $-5^{\circ} \mathrm{C}$. The reaction mixture was allowed to warm to room temperature and then refluxed for $100 \mathrm{~h}$. The solvent was removed by reduced pressure to leave a fine microcrystalline mass which was recrystallised from hot ethanol. The resulting crystals were isolated by filtration, washed with ethanol and air-dried. The esters were used as the precursors for the synthesis of the carboxylate ligands. All esters were fully characterised by ${ }^{1} \mathrm{H},{ }^{13} \mathrm{C}$ NMR and IR spectroscopies, as well as by elemental analyses, melting point and TLC analysis (given as Supplementary data in Tables S1-S3).

\subsubsection{Synthesis of substituted coumarin acids $\mathbf{2 a}, \mathbf{2 b}$ and $\mathbf{2 d - 2 i}$}

The coumarin acids $\mathbf{2 a}, \mathbf{2} \mathbf{b}$ and $\mathbf{2 d}-\mathbf{2} \mathbf{i}$ were synthesised using the following general procedure. The appropriate ester $(1 \mathrm{mmol})$, sodium hydroxide solution $(2 \mathrm{M}, 10 \mathrm{ml})$ and ethanol $(95 \%, 5 \mathrm{ml})$ were heated under reflux for $2 \mathrm{~h}$. After cooling, concentrated hydrochloric acid (37\%) was added drop wise to the solution until a precipitate formed. The resulting solid was isolated by filtration, washed with water and dried in a vacuum oven until a constant mass was obtained.

\subsubsection{Synthesis of 8-methoxycoumarin-4-carboxylic acid [8- \\ $\left.\mathrm{OCH}_{3} \mathrm{CAH}\right](2 \mathrm{c})$}

Methyl-8-methoxy-coumarin-4-carboxylate $(0.7 \mathrm{~g}, 3 \mathrm{mmol})$, $20 \mathrm{ml}$ of hot water and concentrated hydrochloric acid (37\%, $2 \mathrm{ml}$ ) were added to a round bottom flask. The coumarin did not dissolve and the resulting suspension was refluxed with stirring for $11 \mathrm{~h}$. The resulting yellow solid was isolated by filtration, washed first with water $(20 \mathrm{ml})$ and then with ethanol $(20 \mathrm{ml})$ and dried in a vacuum oven until a constant mass was obtained.

\subsubsection{Synthesis of 6-hydroxycoumarin-4-carboxylic acid [6-OHCAH]} (2j), 7-hydroxycoumarin-4-carboxylic acid [7-OHCAH] (2k) and 8hydoxycoumarin-4-carboxylic acid [8-OHCAH] (2l)

6-methoxycoumarin-4-carboxylic acid (2a) $(0.44 \mathrm{~g}, 2 \mathrm{mmol})$ and pyridine hydrochloride $(3 \mathrm{~g}, 26 \mathrm{mmol})$ were irradiated at $200{ }^{\circ} \mathrm{C}$ for $2 \mathrm{~min}$ in a microwave synthesiser. The vessel was cooled to room temperature and irradiated once more for a further $2 \mathrm{~min}$. To this, ice-cold water $(10 \mathrm{ml})$ was added, followed by concentrated hydrochloric acid $(1 \mathrm{M}, 10 \mathrm{ml})$. The reaction mixture was then extracted with ethyl acetate $(40 \mathrm{ml} \times 2)$ using a separating funnel. The resulting organic layer was washed with water $(20 \mathrm{ml} \times 2)$, dried over sodium sulphate and the solvent was removed by reduced pressure. A dark yellow solid $(\mathbf{2 j})$ was obtained. The carboxylate derivatives 7-hydroxycoumarin-4-carboxylic acid 
Table 1

Ligand names, their substituents and positions of R groups for compounds (2a-1).

\begin{tabular}{|c|c|c|c|c|}
\hline \multirow[t]{2}{*}{ Ligand name } & \multirow[t]{2}{*}{ Ligand/molecular formula } & \multicolumn{3}{|c|}{$\mathrm{R}$ groups } \\
\hline & & $\mathrm{R}_{6}$ & $\mathrm{R}_{7}$ & $\mathrm{R}_{8}$ \\
\hline 6-Methoxycoumarin-4-carboxylic acid & 6- $\mathrm{OCH}_{3} \mathrm{CAH}(\mathbf{2 a}) / \mathrm{C}_{11} \mathrm{H}_{8} \mathrm{O}_{5}$ & $\mathrm{OCH}_{3}$ & $\mathrm{H}$ & $\mathrm{H}$ \\
\hline 7-Methoxycoumarin-4-carboxylic acid & 7- $\mathrm{OCH}_{3} \mathrm{CAH}(\mathbf{2 b}) / \mathrm{C}_{11} \mathrm{H}_{8} \mathrm{O}_{5}$ & $\mathrm{H}$ & $\mathrm{OCH}_{3}$ & $\mathrm{H}$ \\
\hline 8-Methoxycoumarin-4-carboxylic acid & $8-\mathrm{OCH}_{3} \mathrm{CAH}(\mathbf{2 c}) / \mathrm{C}_{11} \mathrm{H}_{8} \mathrm{O}_{5}$ & $\mathrm{H}$ & $\mathrm{H}$ & $\mathrm{OCH}_{3}$ \\
\hline 6-Chlorocoumarin-4-carboxylic acid & 6-ClCAH (2d) $/ \mathrm{C}_{10} \mathrm{H}_{5} \mathrm{ClO}_{4}$ & $\mathrm{Cl}$ & $\mathrm{H}$ & $\mathrm{H}$ \\
\hline 6-Bromocoumarin-4-carboxylic acid & 6-BrCAH $(\mathbf{2 e}) / \mathrm{C}_{10} \mathrm{H}_{5} \mathrm{BrO}_{4}$ & $\mathrm{Br}$ & $\mathrm{H}$ & $\mathrm{H}$ \\
\hline 8-Chloro-6-methoxycoumarin-4-carboxylic acid & 8-Cl-6-OCH ${ }_{3} \mathrm{CAH}(\mathbf{2 f}) / \mathrm{C}_{11} \mathrm{H}_{7} \mathrm{ClO}_{5}$ & $\mathrm{OCH}_{3}$ & $\mathrm{H}$ & $\mathrm{Cl}$ \\
\hline 6-Chloro-8-methoxycoumarin-4-carboxylic acid & 6-Cl-8-OCH ${ }_{3} \mathrm{CAH}(\mathbf{2 g}) / \mathrm{C}_{11} \mathrm{H}_{7} \mathrm{ClO}_{5}$ & $\mathrm{Cl}$ & $\mathrm{H}$ & $\mathrm{OCH}_{3}$ \\
\hline 7,8-Dichlorocoumarin-4-carboxylic acid & $7,8-\mathrm{Cl}_{2} \mathrm{CAH}(\mathbf{2 h}) / \mathrm{C}_{10} \mathrm{H}_{4} \mathrm{Cl}_{2} \mathrm{O}_{4}$ & $\mathrm{H}$ & $\mathrm{Cl}$ & $\mathrm{Cl}$ \\
\hline 8-Flouro-6-bromocoumarin-4-carboxylic acid & 8-F-6-BrCAH $(\mathbf{2 i}) / \mathrm{C}_{10} \mathrm{H}_{4} \mathrm{BrFO}_{4}$ & $\mathrm{Br}$ & $\mathrm{H}$ & $\mathrm{F}$ \\
\hline 6-Hydroxycoumarin-4-carboxylic acid & 6-OHCAH $(\mathbf{2} \mathbf{j}) / \mathrm{C}_{10} \mathrm{H}_{6} \mathrm{O}_{5}$ & $\mathrm{OH}$ & $\mathrm{H}$ & $\mathrm{H}$ \\
\hline 7-Hydroxycoumarin-4-carboxylic acid & 7-OHCAH $(\mathbf{2 k}) / \mathrm{C}_{10} \mathrm{H}_{6} \mathrm{O}_{5}$ & $\mathrm{H}$ & $\mathrm{OH}$ & $\mathrm{H}$ \\
\hline 8-Hydroxycoumarin-4-carboxylic acid & 8-ОНСАH $(\mathbf{2 l}) / \mathrm{C}_{10} \mathrm{H}_{6} \mathrm{O}_{5}$ & $\mathrm{H}$ & $\mathrm{H}$ & $\mathrm{OH}$ \\
\hline
\end{tabular}

$\mathrm{CAH}=$ coumarin-4-carboxylic acid.

[7-OHCAH] (2k) and 8-hydoxycoumarin-4-carboxylic acid [8-OH$\mathrm{CAH}$ ( $\mathbf{2 l})$ were synthesised by the same method as that employed to prepare (2a) except compounds ( $2 \mathbf{b})$ and $(\mathbf{2 c})$, respectively were used as the starting reagent.

All ligands were fully characterised by ${ }^{1} \mathrm{H},{ }^{13} \mathrm{C}$ NMR, IR and UVVis spectroscopies, as well as by elemental analyses, melting point and TLC analysis (Tables 2-5).

\subsection{Synthesis of substituted coumarin-4-carboxylatosilver(I) complexes (3a-3l)}

Syntheses of the $\operatorname{Ag}(\mathrm{I})$ complexes were conducted in the absence of light and all complexes were stored in the dark. The $\mathrm{Ag}(\mathrm{I})$ complexes were prepared by the following general method: coumarin acid ( $1 \mathrm{mmol}$ ) was heated with stirring in a aqueous solution of sodium hydroxide $(0.04 \mathrm{~g}, 1 \mathrm{mmol}$ in $10 \mathrm{ml}$ of water). After cooling the resultant suspension to room temperature, sil$\operatorname{ver}(\mathrm{I})$ nitrate solution $(0.170 \mathrm{~g}, 1 \mathrm{mmol}$ in water $(10 \mathrm{ml}))$ was added to it, drop wise over $10 \mathrm{~min}$. The suspension was then stirred for $1 \mathrm{~h}$ and the resulting precipitate was isolated by filtration, washed with hot ethanol $(20 \mathrm{ml})$ and then with cold water $(20 \mathrm{ml})$. The solid was then air-dried in the dark. Analytical data for the $\mathrm{Ag}(\mathrm{I})$ complexes is given in Tables 6-9.

\subsection{Synthesis of 6-chlorocoumarin-4-carboxylatobis(phenanthro} line)silver(I).3.5 $\mathrm{H}_{2} \mathrm{O}(\mathbf{4 d})$, 8-chloro-6-methoxycoumarin-4-carboxy lato(phenanthroline)silver(I) (4f) and 6-chloro-8-methoxycoumarin4-carboxylatebis(phenanthroline)silver(I) $3.5 \mathrm{H}_{2} \mathrm{O}(\mathbf{4 g})$

The $\mathrm{Ag}(\mathrm{I})$ phenanthroline adducts ( $\mathbf{4 d}, \mathbf{4 f}$ and $\mathbf{4 g}$ ) were prepared by the following general method: coumarin acid $(0.5 \mathrm{mmol})$ and

\section{Table 2}

Analytical data for the coumarin carboxylate ligands (2a-1).

\begin{tabular}{|c|c|c|c|c|c|c|c|c|}
\hline \multirow[t]{2}{*}{ Ligand } & \multirow[t]{2}{*}{$\mathrm{MW}(\mathrm{g} / \mathrm{mol})$} & \multirow[t]{2}{*}{ Yield (\%) } & \multirow[t]{2}{*}{ Empirical formula } & \multicolumn{2}{|c|}{ Found (Calc.) (\%) } & \multirow[t]{2}{*}{ M.P $\left({ }^{\circ} \mathrm{C}\right)^{\mathrm{a}}$} & \multirow[t]{2}{*}{$\mathrm{R}_{\mathrm{f}}^{\mathrm{b}}$} & \multirow[t]{2}{*}{$\Lambda_{\mathrm{M}}\left(\mathrm{S} \mathrm{cm}^{2} \mathrm{~mol}^{-1}\right)^{\mathrm{c}}$} \\
\hline & & & & $\mathrm{C}$ & $\mathrm{H}$ & & & \\
\hline $6-\mathrm{OCH}_{3} \mathrm{CAH}(\mathbf{2 a})$ & 220.18 & 64 & $\mathrm{C}_{11} \mathrm{H}_{8} \mathrm{O}_{5}$ & $59.94(60.00)$ & $3.66(3.66)$ & $246-248$ & 0.66 & 0.49 \\
\hline 7- $\mathrm{OCH}_{3} \mathrm{CAH}(\mathbf{2 b})$ & 220.18 & 84 & $\mathrm{C}_{11} \mathrm{H}_{8} \mathrm{O}_{5}$ & $59.90(60.00)$ & $3.55(3.66)$ & $223-225$ & 0.83 & 0.48 \\
\hline $8-\mathrm{OCH}_{3} \mathrm{CAH}(\mathbf{2 c})$ & 220.18 & 77 & $\mathrm{C}_{11} \mathrm{H}_{8} \mathrm{O}_{5}$ & $59.74(60.00)$ & $3.57(3.66)$ & $229-232$ & 0.67 & 0.40 \\
\hline 6-ClCAH (2d) & 224.60 & 80 & $\mathrm{C}_{10} \mathrm{H}_{5} \mathrm{ClO}_{4}$ & $52.98(53.48)$ & $2.16(2.24)$ & $230-232$ & 0.75 & 0.45 \\
\hline $6-\mathrm{BrCAH}(\mathbf{2 e})$ & 269.05 & 67 & $\mathrm{C}_{10} \mathrm{H}_{5} \mathrm{BrO}_{4}$ & $44.48(44.64)$ & $1.84(1.87)$ & $240-242$ & 0.71 & 0.45 \\
\hline $8-\mathrm{Cl}-6-\mathrm{OCH}_{3} \mathrm{CAH}(\mathbf{2 f})$ & 254.62 & 79 & $\mathrm{C}_{11} \mathrm{H}_{7} \mathrm{ClO}_{5}$ & $51.70(51.89)$ & $2.74(2.77)$ & $282-284$ & 0.73 & 0.56 \\
\hline $6-\mathrm{Cl}-8-\mathrm{OCH}_{3} \mathrm{CAH}(\mathbf{2 g})$ & 254.62 & 94 & $\mathrm{C}_{11} \mathrm{H}_{7} \mathrm{ClO}_{5}$ & $51.64(51.89)$ & $3.06(2.77)$ & $244-246$ & 0.78 & 0.94 \\
\hline 7,8-Cl ${ }_{2} \mathrm{CAH}(\mathbf{2 h})$ & 263.07 & 97 & $\mathrm{C}_{10} \mathrm{H}_{4} \mathrm{Cl}_{2} \mathrm{O}_{4}$ & $45.62(46.37)$ & $1.45(1.56)$ & $249-250$ & 0.75 & 0.73 \\
\hline 8-F-6-BrCAH (2i) & 287.04 & 71 & $\mathrm{C}_{10} \mathrm{H}_{4} \mathrm{BrFO}_{4}$ & $41.73(41.84)$ & $1.30(1.40)$ & $232-233$ & 0.84 & 1.60 \\
\hline $6-\mathrm{OHCAH} \mathrm{(2 \mathbf {j } )}$ & 206.15 & 77 & $\mathrm{C}_{10} \mathrm{H}_{6} \mathrm{O}_{5}$ & $58.35(58.26)$ & $3.05(2.93)$ & $286-288$ & 0.75 & 0.46 \\
\hline 7-ОНСАН (2k) & 206.15 & 74 & $\mathrm{C}_{10} \mathrm{H}_{6} \mathrm{O}_{5}$ & $58.38(58.26)$ & $3.13(2.93)$ & $251-253$ & 0.72 & 0.51 \\
\hline 8-OHCAH (21) & 206.15 & 77 & $\mathrm{C}_{10} \mathrm{H}_{6} \mathrm{O}_{5}$ & $58.17(58.26)$ & $3.01(2.93)$ & $247-249$ & 0.75 & 0.52 \\
\hline
\end{tabular}

\footnotetext{
${ }^{\text {a }}$ M.P.s are uncorrected.

b Mobile phase 50:50 ethyl acetate:methanol.

c Recorded in DMSO $\left(4 \times 10^{-3} \mathrm{M}\right)$.
}

sodium hydroxide solution $(0.02 \mathrm{~g}, 0.5 \mathrm{mmol}$ in water $(10 \mathrm{ml})$ ) were stirred for $30 \mathrm{~min}$. A silver nitrate solution $(0.085 \mathrm{~g}, 0.5 \mathrm{mmol}$ in water $(10 \mathrm{ml})$ ) was added drop wise to the coumarin solution, the resultant yellow suspension was stirred for $1 \mathrm{~h}$. 1,10-Phenanthroline $(0.180 \mathrm{~g}, 1 \mathrm{mmol})$ dissolved in ethanol $(10 \mathrm{ml})$ was then added and the reaction mixture was stirred for a further $2 \mathrm{~h}$. A yellow solid was isolated by filtration, washed with water $(30 \mathrm{ml})$, methanol $(20 \mathrm{ml})$ and allowed to air-dry. Analytical data for the complexes is given in Tables 6-9.

\subsection{Antimicrobial studies}

The ligands (2a-1), $\mathrm{Ag}(\mathrm{I})$ complexes (3a-1), phenanthroline adducts (4d, $\mathbf{4 f}$ and $\mathbf{4 g}$ ) and commercially available drugs gentamycin and vancomycin were screened for their antimicrobial activity. E. coli (NCIMB 9485), S. aureus (NCIMB 12702) were obtained from the National Collection of Industrial, Marine and Food. MRSA (ATCC4300) and P. aeruginosa strains (PA01 and ATCC 27853) were obtained from the American Type Culture Collection. E. coli and $P$. aeruginosa were cultured in Luria Bertani (LB) broth (Sigma) while S. aureus was cultured in Tryptone Soy Broth (TSB, LabM, UK). MRSA was cultured in nutrient broth. Bacteria were stored in broth with $20 \%$ glycerol at $-80{ }^{\circ} \mathrm{C}$, and passaged twice on agar plate (LB agar, TSA or nutrient agar respectively) before being used in an experiment. Minimum inhibitory concentrations (MIC) were determined by broth microdilution. Stock solutions of each compound to be tested were prepared in DMSO and serially diluted in triplicate using broth $(100 \mu \mathrm{l} /$ well $)$ at a concentration range of $0.195-$ $200 \mu \mathrm{M}$ on microtitre plates, after which the final concentration of DMSO in the cell suspension was not greater than $1 \%$. Overnight cultures $(10 \mathrm{ml})$ of bacterial strains were inoculated in broth 
Table 3

Selected IR and UV-Vis data for the coumarin ligands (2a-1).

\begin{tabular}{|c|c|c|c|c|c|}
\hline \multirow[t]{2}{*}{ Ligand } & \multicolumn{3}{|c|}{$\operatorname{IR}\left(\mathrm{cm}^{-1}\right)^{\mathrm{a}}$} & \multicolumn{2}{|l|}{ UV-Vis ${ }^{b}$} \\
\hline & $v_{\mathrm{OH}}$ & $v_{\mathrm{C}=\mathrm{O}}$ (lactone) & $v_{\mathrm{C}=\mathrm{O}}($ acid $)$ & $\lambda_{\max }(\mathrm{nm})$ & $\varepsilon\left(\mathrm{M}^{-1} \mathrm{~cm}^{-1}\right)$ \\
\hline \multirow[t]{2}{*}{$6-\mathrm{OCH}_{3} \mathrm{CAH}(\mathbf{2 a})$} & 2924 & 1720 & 1681 & 285 & 7457 \\
\hline & & & & 354 & 3000 \\
\hline 7- $\mathrm{OCH}_{3} \mathrm{CAH}(\mathbf{2 b})$ & 2972 & 1732 & 1676 & 336 & 11650 \\
\hline $8-\mathrm{OCH}_{3} \mathrm{CAH}(\mathbf{2 c})$ & 2948 & 1737 & 1677 & 296 & 8525 \\
\hline \multirow[t]{2}{*}{ 6-ClCAH (2d) } & 2909 & 1726 & 1691 & 280 & 8225 \\
\hline & & & & $327^{c}$ & $4525^{c}$ \\
\hline \multirow[t]{2}{*}{ 6-BrCAH (2e) } & 2889 & 1727 & 1686 & 281 & 7000 \\
\hline & & & & $328^{c}$ & $3600^{c}$ \\
\hline \multirow[t]{2}{*}{$8-\mathrm{Cl}-6-\mathrm{OCH}_{3} \mathrm{CAH}(\mathbf{2 f})$} & 2979 & 1721 & 1693 & 288 & 8575 \\
\hline & & & & 355 & 2750 \\
\hline $6-\mathrm{Cl}-8-\mathrm{OCH}_{3} \mathrm{CAH}(\mathbf{2 g})$ & 2924 & 1719 & 1697 & 295 & 7175 \\
\hline $7,8-\mathrm{Cl}_{2} \mathrm{CAH}(\mathbf{2 h})$ & 2930 & 1732 & 1702 & 294 & 7575 \\
\hline 8-F-6-BrCAH (2i) & 3170 & 1749 & 1721 & 285 & 7950 \\
\hline \multirow[t]{2}{*}{ 6-OHCAH (2j) } & 3079 & 1736 & 1681 & 290 & 8200 \\
\hline & & & & 365 & 3250 \\
\hline 7-ОНСАН (2k) & 3114 & - & 1697 & 340 & 8625 \\
\hline 8-ОНСАН (21) & 3093 & 1734 & 1689 & 305 & 9500 \\
\hline
\end{tabular}

a All IR data was recorded as $\mathrm{KBr}$ discs.

b All UV-Vis data was recorded in DMSO.

c Shoulder.

Table 4

${ }^{1} \mathrm{H}$ NMR data for coumarin-4-carboxylic acids recorded in $\mathrm{d}_{6}$-DMSO, showing signal in ppm, multiplicity and $J$ values in $\mathrm{Hz}$.

\begin{tabular}{|c|c|c|c|c|c|c|}
\hline Ligand & H4 (vinyl) & H5 & $\mathrm{H} 6$ & $\mathrm{H} 7$ & $\mathrm{H} 8$ & $\mathrm{COOH}$ \\
\hline $6-\mathrm{OCH}_{3} \mathrm{CAH}(\mathbf{2 a})$ & $6.85, \mathrm{~s}$ & $7.69, \mathrm{~d}, J=2.9$ & $3.79, \mathrm{~s}$ & $7.28, \mathrm{dd}, J=2.9,9.0$ & $7.41, \mathrm{~d}, J=9.0$ & $14.48(\mathrm{OH})$ \\
\hline $7-\mathrm{OCH}_{3} \mathrm{CAH}(\mathbf{2 b})$ & $6.66, \mathrm{~s}$ & $8.09, \mathrm{~d}, J=8.9$ & $7.01, \mathrm{dd}, J=2.5,8.9$ & $3.81, \mathrm{~s}$ & $7.10, \mathrm{~d}, J=2.5$ & \\
\hline $8-\mathrm{OCH}_{3} \mathrm{CAH}(\mathbf{2 c})$ & $6.83, \mathrm{~s}$ & $7.65, \mathrm{dd}, J=1.8,7.7$ & $7.29, \mathrm{pt}, J=7.7,8.2$ & $7.34, \mathrm{dd}, J=1.8,8.2$ & $3.92, \mathrm{~s}$ & $14.36(\mathrm{OH})$ \\
\hline 6-ClCAH (2d) & $6.95, \mathrm{~s}$ & $8.30, \mathrm{~d}, J=2.6$ & $\mathrm{Cl}$ & $7.71, \mathrm{dd}, J=2.6,8.8$ & $7.49, \mathrm{~d}, J=8.8$ & \\
\hline $6-\mathrm{BrCAH}(\mathbf{2 e})$ & $6.99, \mathrm{~s}$ & $8.45, \mathrm{~d}, J=2.4$ & $\mathrm{Br}$ & $7.87, \mathrm{dd}, J=2.4,8.8$ & $7.48, \mathrm{~d}, J=8.8$ & \\
\hline $8-\mathrm{Cl}-6-\mathrm{OCH}_{3} \mathrm{CAH}(\mathbf{2 f})$ & $6.99, \mathrm{~s}$ & $7.74, \mathrm{~d}, J=2.9$ & $3.86, \mathrm{~s}$ & $7.56, \mathrm{~d}, J=2.9$ & $\mathrm{Cl}$ & $14.43(\mathrm{OH})$ \\
\hline $6-\mathrm{Cl}-8-\mathrm{OCH}_{3} \mathrm{CAH}(\mathbf{2 g})$ & $6.96, \mathrm{~s}$ & $7.80, \mathrm{~d}, J=2.3$ & $\mathrm{Cl}$ & $7.45, \mathrm{~d}, J=2.3$ & $3.94, s$ & \\
\hline 7,8-Cl ${ }_{2} \mathrm{CAH}(\mathbf{2 h})$ & $6.97, \mathrm{~s}$ & $8.16, \mathrm{~d}, J=8.9$ & $\begin{array}{l}7.66, \mathrm{~d} \\
J=8.9\end{array}$ & $\mathrm{Cl}$ & $\mathrm{Cl}$ & \\
\hline 8-F-6-BrCAH (2i) & $7.07, \mathrm{~s}$ & $8.28, \mathrm{t}, J=2$ & $\mathrm{Br}$ & $8.06, \mathrm{dd}, J=2,10$ & $\mathrm{~F}$ & \\
\hline $6-\mathrm{OHCAH}(\mathbf{2} \mathbf{j})$ & $6.81, \mathrm{~s}$ & $7.57, \mathrm{~d}, J=2.9$ & $9.82(\mathrm{OH})$ & $7.08, \mathrm{dd}, J=2.9,8.9$ & $7.31, \mathrm{~d}, J=8.9$ & $14.30(\mathrm{OH})$ \\
\hline 7-ОНСAH (2k) & $6.57, \mathrm{~s}$ & $7.98, \mathrm{~d}, J=8.8$ & $6.84, \mathrm{dd}, J=2.4,8.8$ & $10.73(\mathrm{OH})$ & $6.77, \mathrm{~d}, J=2.4$ & $14.29(\mathrm{OH})$ \\
\hline $8-\mathrm{OHCAH} \mathrm{(21)}$ & $6.80, \mathrm{~s}$ & $7.53, \mathrm{dd}, J=2.1,8.0$ & $7.20, \mathrm{pt}, J=8.0$ & $7.15, \mathrm{dd}, J=2.1,8.0$ & $10.33(\mathrm{OH})$ & \\
\hline
\end{tabular}

Table 5

${ }^{13} \mathrm{C}$ NMR data (ppm) for coumarin-4-carboxylic acids recorded in $\mathrm{d}_{6}$-DMSO.

\begin{tabular}{|c|c|c|c|c|c|c|c|c|c|c|c|}
\hline Ligand & C2 (lactone) & C3 (vinyl) & $\mathrm{C} 4$ & C5 & C6 & $\mathrm{C} 7$ & $\mathrm{C} 8$ & C9 & $\mathrm{C} 10$ & C11 (carbonyl) & $\mathrm{OCH}_{3}$ \\
\hline $6-\mathrm{OCH}_{3} \mathrm{CAH}(\mathbf{2 a})$ & 159.8 & 118.0 & 143.5 & 109.5 & 155.6 & 119.6 & 118.6 & 148.2 & 116.2 & 165.4 & 55.7 \\
\hline $7-\mathrm{OCH}_{3} \mathrm{CAH}(\mathbf{2 b})$ & 162.6 & 114.1 & 144.1 & 127.7 & 112.7 & 159.9 & 101.0 & 155.7 & 109.1 & 165.5 & 56 \\
\hline $8-\mathrm{OCH}_{3} \mathrm{CAH}(\mathbf{2 c})$ & 159.3 & 117.7 & 144.6 & 116.3 & 124.6 & 114.6 & 146.8 & 143.2 & 117.7 & 165.5 & 56.2 \\
\hline 6-CICAH (2d) & 159.2 & 119.8 & 142.2 & 126.0 & 128.5 & 131.9 & 118.8 & 152.4 & 117.3 & 164.9 & - \\
\hline 6-BrCAH (2e) & 159.2 & 119.9 & 142.0 & 129.0 & 116.4 & 134.8 & 119.2 & 152.9 & 117.7 & 165.0 & - \\
\hline $8-\mathrm{Cl}-6-\mathrm{OCH}_{3} \mathrm{CAH}(\mathbf{2} \mathbf{f})$ & 158.6 & 119.6 & 142.9 & 109.5 & 155.1 & 119.0 & 120.8 & 143.6 & 117.6 & 165.0 & 56.0 \\
\hline $6-\mathrm{Cl}-8-\mathrm{OCH}_{3} \mathrm{CAH}(\mathbf{2 g})$ & 158.8 & 119.8 & 142.5 & 116.8 & 128.5 & 114.6 & 147.6 & 142.3 & 117.1 & 163.7 & 56.7 \\
\hline $7,8-\mathrm{Cl}_{2} \mathrm{CAH}(\mathbf{2 h})$ & 158.4 & 116.2 & 142.8 & 126.0 & 125.5 & 135.1 & 119.3 & 150.4 & 118.9 & 164.8 & - \\
\hline $8-\mathrm{F}-6-\mathrm{BrCAH}(\mathbf{2 i})$ & 157.9 & 120.9 & $141.6^{\mathrm{a}}$ & $124.7^{\mathrm{b}}$ & $115.2^{\mathrm{c}}$ & $121.4^{\mathrm{d}}$ & $148.8^{\mathrm{e}}$ & $141.5^{\mathrm{f}}$ & $119.2^{g}$ & 164.6 & - \\
\hline 6-OHCAH (2j) & 159.7 & 117.6 & 143.7 & 110.9 & 153.8 & 120.3 & 117.8 & 147.0 & 116.1 & 165.4 & - \\
\hline 7-OHCAH (2k) & 160.1 & 112.9 & 144.4 & 128.1 & 113.4 & 161.5 & 102.6 & 155.8 & 107.9 & 165.4 & - \\
\hline $8-\mathrm{OHCAH}(\mathbf{2 1})$ & 159.4 & 117.2 & 144.9 & 116.5 & 124.5 & 118.6 & 144.9 & 142.5 & 116.5 & 165.5 & - \\
\hline
\end{tabular}

$\mathrm{d}, J_{\mathrm{C}-\mathrm{F}}=2.4$

b $J_{C-F}=3.6$.

c $\mathrm{d}, J_{\mathrm{C}-\mathrm{F}}=8.5$

d $\mathrm{d}, J_{\mathrm{C}-\mathrm{F}}=20.4$.

e d, $J_{\mathrm{C}-\mathrm{F}}=251$.

f $\mathrm{d}, J_{\mathrm{C}-\mathrm{F}}=11.5$

g $\mathrm{d}, J_{\mathrm{C}-\mathrm{F}}=1.8$.

$(100 \mathrm{ml})$ and incubated at $37^{\circ} \mathrm{C}$ with agitation, optical density at $600 \mathrm{~nm}\left(\mathrm{OD}_{600}\right)$ was measured and strains were then diluted to $1 \times 10^{6} \mathrm{CFU} / \mathrm{ml}$ and seeded at $100 \mu \mathrm{l} /$ well in microtitre well plates. Bacteria were also treated with a solvent control (0.5\% DMSO) in control wells. Following $24 \mathrm{~h}$ incubation the absorbance was read in a microtitre plate reader [28].

Each compound was screened at each concentration at least in triplicate and three independent experiments were carried out. 
Table 6

Analytical data for the $\mathrm{Ag}(\mathrm{I})$ complexes $\mathbf{3 a - 1}$ and $\mathbf{4 d}, \mathbf{4 f}$ and $\mathbf{4 g}$.

\begin{tabular}{|c|c|c|c|c|c|c|c|c|c|}
\hline \multirow[t]{2}{*}{ Complex } & \multirow{2}{*}{$\begin{array}{l}\mathrm{MW}(\mathrm{g} / \\
\mathrm{mol})\end{array}$} & \multirow{2}{*}{$\begin{array}{l}\text { Yield } \\
(\%)\end{array}$} & \multirow{2}{*}{$\begin{array}{l}\text { Empirical } \\
\text { formula }\end{array}$} & \multicolumn{4}{|c|}{ Found (Calc.) (\%) } & \multirow{2}{*}{$\begin{array}{l}\text { M.P } \\
\left({ }^{\circ} \mathrm{C}\right)\end{array}$} & \multirow{2}{*}{$\begin{array}{l}\Lambda_{\mathrm{M}} \\
\left(\mathrm{S} \mathrm{cm}^{2} \mathrm{~mol}^{-1}\right)\end{array}$} \\
\hline & & & & C & $\mathrm{H}$ & $\mathrm{N}$ & $\mathrm{Ag}$ & & \\
\hline$\left[\mathrm{Ag}\left(6-\mathrm{OCH}_{3} \mathrm{CA}\right)\right](\mathbf{3 a})$ & 327.04 & 68 & $\mathrm{C}_{11} \mathrm{H}_{7} \mathrm{AgO}_{5}$ & $\begin{array}{l}39.41 \\
(40.40)\end{array}$ & $\begin{array}{l}2.03 \\
(2.16)\end{array}$ & - & $\begin{array}{l}33.01 \\
(32.98)\end{array}$ & $>300$ & 10.85 \\
\hline$\left[\mathrm{Ag}\left(7-\mathrm{OCH}_{3} \mathrm{CA}\right)\right](\mathbf{3 b})$ & 327.04 & 68 & $\mathrm{C}_{11} \mathrm{H}_{7} \mathrm{AgO}_{5}$ & $\begin{array}{l}39.67 \\
(40.40)\end{array}$ & $\begin{array}{l}2.13 \\
(2.16)\end{array}$ & - & $\begin{array}{l}33.03 \\
(32.98)\end{array}$ & $>300$ & 10.23 \\
\hline$\left[\mathrm{Ag}\left(8-\mathrm{OCH}_{3} \mathrm{CA}\right)\right](3 \mathrm{c})$ & 327.04 & 57 & $\mathrm{C}_{11} \mathrm{H}_{7} \mathrm{AgO}_{5}$ & $\begin{array}{l}40.22 \\
(40.40)\end{array}$ & $\begin{array}{l}2.27 \\
(2.16)\end{array}$ & - & $\begin{array}{l}32.60 \\
(32.98)\end{array}$ & $\begin{array}{l}243- \\
246\end{array}$ & 10.10 \\
\hline$[\mathrm{Ag}(6-\mathrm{ClCA})](\mathbf{3 d})$ & 331.46 & 63 & $\mathrm{C}_{10} \mathrm{H}_{4} \mathrm{ClAgO}_{4}$ & $\begin{array}{l}36.05 \\
(36.24)\end{array}$ & $\begin{array}{l}1.19 \\
(1.22)\end{array}$ & - & $\begin{array}{l}31.92 \\
(32.54)\end{array}$ & $>300$ & 12.55 \\
\hline$[\mathrm{Ag}(6-\mathrm{BrCA})](\mathbf{3 e})$ & 375.91 & 76 & $\mathrm{C}_{10} \mathrm{H}_{4} \mathrm{AgBrO}_{4}$ & $\begin{array}{l}31.73 \\
(31.95)\end{array}$ & $\begin{array}{l}1.15 \\
(1.07)\end{array}$ & - & $\begin{array}{l}28.13 \\
(28.70)\end{array}$ & $>300$ & 15.71 \\
\hline$\left[\mathrm{Ag}\left(8-\mathrm{Cl}-6-\mathrm{OCH}_{3} \mathrm{CA}\right)\right](\mathbf{3 f})$ & 361.48 & 93 & $\mathrm{C}_{11} \mathrm{H}_{6} \mathrm{AgClO}_{5}$ & $\begin{array}{l}36.55 \\
(36.55)\end{array}$ & $\begin{array}{l}1.67 \\
(1.67)\end{array}$ & - & $\begin{array}{l}29.51 \\
(29.84)\end{array}$ & $>300$ & 11.42 \\
\hline$\left[\mathrm{Ag}\left(6-\mathrm{Cl}-8-\mathrm{OCH}_{3} \mathrm{CA}\right)\right](\mathbf{3 g})$ & 361.48 & 66 & $\mathrm{C}_{11} \mathrm{H}_{6} \mathrm{AgClO}_{5}$ & $\begin{array}{l}37.40 \\
(36.55)\end{array}$ & $\begin{array}{l}1.80 \\
(1.67)\end{array}$ & - & $\begin{array}{l}29.76 \\
(29.84)\end{array}$ & $>300$ & 11.13 \\
\hline$\left[\mathrm{Ag}\left(7,8-\mathrm{Cl}_{2} \mathrm{CA}\right)\right](\mathbf{3 h})$ & 365.90 & 77 & $\mathrm{C}_{10} \mathrm{H}_{3} \mathrm{AgCl}_{2} \mathrm{O}_{4}$ & $\begin{array}{l}32.14 \\
(32.82)\end{array}$ & $\begin{array}{l}0.67 \\
(0.83)\end{array}$ & - & $\begin{array}{l}28.90 \\
(29.48)\end{array}$ & $>300$ & 10.87 \\
\hline$[\mathrm{Ag}(8-\mathrm{F}-6-\mathrm{BrCA})](\mathbf{3 i})$ & 393.90 & 87 & $\mathrm{C}_{10} \mathrm{H}_{3} \mathrm{AgBrFO}_{4}$ & $\begin{array}{l}30.39 \\
(30.49)\end{array}$ & $\begin{array}{l}0.60 \\
(0.77)\end{array}$ & - & $\begin{array}{l}26.91 \\
(27.38)\end{array}$ & $>300$ & 13.73 \\
\hline$[\mathrm{Ag}(6-\mathrm{OHCA})](\mathbf{3} \mathbf{j})$ & 313.01 & 64 & $\mathrm{C}_{10} \mathrm{H}_{5} \mathrm{AgO}_{5}$ & $\begin{array}{l}37.95 \\
(38.37)\end{array}$ & $\begin{array}{l}1.93 \\
(1.61)\end{array}$ & - & $\begin{array}{l}34.39 \\
(34.46)\end{array}$ & $>300$ & 8.88 \\
\hline$[\mathrm{Ag}(7-\mathrm{OHCA})](\mathbf{3 k})$ & 313.01 & 64 & $\mathrm{C}_{10} \mathrm{H}_{5} \mathrm{AgO}_{5}$ & $\begin{array}{l}38.10 \\
(38.37)\end{array}$ & $\begin{array}{l}1.59 \\
(1.61)\end{array}$ & - & $\begin{array}{l}33.90 \\
(34.46)\end{array}$ & $>300$ & 8.78 \\
\hline$[\mathrm{Ag}(8-\mathrm{OHCA})](\mathbf{3 1})$ & 313.01 & 32 & $\mathrm{C}_{10} \mathrm{H}_{5} \mathrm{AgO}_{5}$ & $\begin{array}{l}38.03 \\
(38.37)\end{array}$ & $\begin{array}{l}2.01 \\
(1.61)\end{array}$ & - & $\begin{array}{l}33.94 \\
(34.46)\end{array}$ & $>300$ & 8.81 \\
\hline$\left[\mathrm{Ag}(\text { phen })_{2}(6-\mathrm{ClCA})\right] \cdot 3.5 \mathrm{H}_{2} \mathrm{O}(\mathbf{4 d})$ & 754.92 & 33 & $\mathrm{C}_{34} \mathrm{H}_{27} \mathrm{AgClN}_{4} \mathrm{O}_{7.5}$ & $\begin{array}{l}53.65 \\
(54.09)\end{array}$ & $\begin{array}{l}2.80 \\
(3.60)\end{array}$ & $\begin{array}{l}7.99 \\
(7.42)\end{array}$ & $\begin{array}{l}13.54 \\
(14.29)\end{array}$ & $\begin{array}{l}227- \\
229\end{array}$ & - \\
\hline$\left[\mathrm{Ag}(\right.$ phen $\left.)\left(8-\mathrm{Cl}-6-\mathrm{OCH}_{3} \mathrm{CA}\right)\right](\mathbf{4 f})$ & 541.69 & 63 & $\mathrm{C}_{23} \mathrm{H}_{14} \mathrm{AgClN}_{2} \mathrm{O}_{5}$ & $\begin{array}{l}50.77 \\
(51.00)\end{array}$ & $\begin{array}{l}2.38 \\
(2.61)\end{array}$ & $\begin{array}{l}5.22 \\
(5.17)\end{array}$ & $\begin{array}{l}18.93 \\
(19.19)\end{array}$ & $\begin{array}{l}227- \\
230\end{array}$ & - \\
\hline $\begin{array}{l}{\left[\mathrm{Ag}(\text { phen })_{2}\left(6-\mathrm{Cl}-8-\mathrm{OCH}_{3} \mathrm{CA}\right)\right] \cdot 3.5 \mathrm{H}_{2} \mathrm{O}} \\
\quad(\mathbf{4 g})\end{array}$ & 784.95 & 34 & $\mathrm{C}_{35} \mathrm{H}_{29} \mathrm{AgClN}_{4} \mathrm{O}_{8.5}$ & $\begin{array}{l}53.42 \\
(53.55)\end{array}$ & $\begin{array}{l}3.47 \\
(3.72)\end{array}$ & $\begin{array}{l}6.97 \\
(7.14)\end{array}$ & $\begin{array}{l}14.15 \\
(13.74)\end{array}$ & $\begin{array}{l}226- \\
227\end{array}$ & - \\
\hline
\end{tabular}

The $\mathrm{MIC}_{90}$ values were determined as the concentration of test compound which inhibited 90\% visible growth in $24 \mathrm{~h}$.

\subsubsection{Biofilm assay}

Strains were cultured in Cation adjusted Mueller Hinton broth (CAMHB) overnight and inoculated in fresh CAMHB to reach an OD600 of 0.6. Bacterial biofilms were formed on pegs of a modified polystyrene lid by inoculating $100 \mu \mathrm{l}$ of bacterial suspension $\left(10^{6} \mathrm{CFU} / \mathrm{ml}\right)$ to each well of a polystyrene plate and incubated

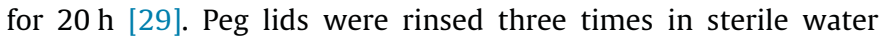
and placed into 96 well plates containing a range of test dilutions in $100 \mu \mathrm{l} \mathrm{CAMHB}$ and incubated for $18 \mathrm{~h}$. The peg lids were placed $0.5 \%$ crystal violet solution for $15 \mathrm{~min}$, rinsed and dried for several hours. The pegs were then incubated in $95 \%$ ethanol with $0.05 \%$ Triton X 100 for $15 \mathrm{~min}$ and absorbance measured at $590 \mathrm{~nm}$. The $\mathrm{BIC}_{90}$ values, biofilm inhibitory concentration, were determined as the minimal concentration of compound needed to reduce biofilm formation by $90 \%$ after $18 \mathrm{~h}$ treatment.

\section{Results and discussion}

\subsection{Synthesis and characterisation of the ligands}

The carboxylate ligands were synthesised by first preparing their corresponding esters ( $\mathbf{1 a}-\mathbf{i})$ via an aromatic electrophilic substitution reaction between the conjugated base of substituted phenols and a vinyl triphenylphosphonium salt [30]. This reaction which involved refluxing the appropriate phenol with triphenylphosphine and dimethylacetylene dicarboxylate in dichloromethane for $100 \mathrm{~h}$ yielded the starting esters. The products were isolated by recrystallisation from ethanol and characterised by standard techniques. The acid derivatives were then synthesised by either acid or base hydrolysis of the corresponding ester or a demethylation reaction (Scheme 1). The acid derivative $\left[8-\mathrm{OCH}_{3-}\right.$ $\mathrm{CAH}](\mathbf{2 c})$ was synthesised by refluxing the ester in water and $10 \%$ hydrochloric acid solution with an average reaction time of $11 \mathrm{~h}$. Ester 1c was not soluble in the acid/water mixture and remained as a suspension; the suspended product was then isolated by filtration. The acid derivatives $\mathbf{2 a}, \mathbf{2 b}$ and $\mathbf{2 d - i}$ could also be synthesised by this method however better yields were obtained by base hydrolysis. The acid 2c could also be synthesised by base hydrolysis but gave a higher yield when made using acid hydrolysis. Synthesis of the hydroxy substituted acid derivatives $\mathbf{2 j} \mathbf{j}-\mathbf{l}$ was carried out by demethylation reaction of the corresponding methoxy substituted acids Scheme 2. Demethylation was achieved by a microwave-assisted reaction using pyridine hydrochloride followed by quenching with $\mathrm{HCl}$ and ice water; products were extracted using ethyl acetate [31]. Physical data and empirical formulae are presented in Table 2. All of the ligands had good solubility in a range of organic solvents.

IR spectral data for the ligands is given in Table 3. The formation of the coumarin acid derivatives was confirmed by the appearance of the characteristic acid bands, $v_{\mathrm{C}=\mathrm{O}}$ and $v_{\mathrm{OH}}$. There were two $v_{\mathrm{C}=\mathrm{O}}$ bands corresponding to the $v_{\mathrm{C}=\mathrm{O}}$ (acid) and the $v_{\mathrm{C}=\mathrm{O}}$ (lactone) and these were observed at between 1721-1677 and $1720-1737 \mathrm{~cm}^{-1}$ respectively. In a previous study on coumarin-3-carboxylate the lower frequency band was assigned to the lactone carbonyl though in this molecule intramolecular hydrogen bonding between the lactone carbonyl and hydrogen of the acid was thought to lower the frequency of the lactone carbonyl [32]. In the coumarin-4-carboxylate ligands the assignment of the two $v_{\mathrm{C}=\mathrm{O}}$ bands was based on consideration of the lactone as a cyclic ester in which the carbonyl group of $\alpha, \beta$-unsaturated esters occurs at about 1730 $1715 \mathrm{~cm}^{-1}$ and the carbonyl of a $\delta$-lactone (six-membered ring) occurs in the same region as unconjugated esters $\left(1750-1735 \mathrm{~cm}^{-1}\right.$ ) 
Table 7

Selected IR and UV-Vis data for the Ag(I) complexes $\mathbf{3 a - 1}$ and $\mathbf{4 d}, \mathbf{4 f}$ and $\mathbf{4 g}$.

\begin{tabular}{|c|c|c|c|c|c|c|}
\hline \multicolumn{5}{|l|}{$\operatorname{IR}\left(\mathrm{cm}^{-1}\right)^{\mathrm{a}}$} & \multicolumn{2}{|l|}{ UV-Vis ${ }^{\mathrm{b}}$} \\
\hline Complex & $v_{\mathrm{C}=\mathrm{O}}$ & $v_{\text {asym }}(\mathrm{OCO})$ & $v_{\text {sym }}(\mathrm{OCO})$ & $\Delta v(\mathrm{OCO})$ & $\lambda_{\max }(\mathrm{nm})$ & $\varepsilon\left(\mathrm{M}^{-1} \mathrm{~cm}^{-1}\right)$ \\
\hline \multirow{2}{*}[\mathrm{Ag}(6-\mathrm{OCH}_{3}\mathrm{CA})]{$(\mathbf{3 a})$} & 1694 & 1625 & 1402 & 223 & 278 & 10200 \\
\hline & & & & & 340 & 4925 \\
\hline$\left[\mathrm{Ag}\left(7-\mathrm{OCH}_{3} \mathrm{CA}\right)\right](\mathbf{3 b})$ & 1698 & 1618 & 1399 & 219 & 322 & 13350 \\
\hline$\left[\mathrm{Ag}\left(8-\mathrm{OCH}_{3} \mathrm{CA}\right)\right](3 \mathrm{c})$ & 1703 & 1606 & 1395 & 211 & 289 & 14025 \\
\hline \multirow[t]{2}{*}[\mathrm{Ag}(6-\mathrm{ClCA})]{$(\mathbf{3 d})$} & 1704 & 1604 & 1390 & 214 & 274 & 11650 \\
\hline & & & & & 320 & 7425 \\
\hline \multirow[t]{2}{*}[\mathrm{Ag}(6-\mathrm{BrCA})]{$(\mathbf{3 e})$} & 1716 & 1615 & 1411 & 204 & 274 & 10300 \\
\hline & & & & & 321 & 6175 \\
\hline \multirow[t]{2}{*}[\mathrm{Ag}(8-\mathrm{Cl}-6-\mathrm{OCH}_{3}\mathrm{CA})]{$(\mathbf{3 f})$} & 1702 & 1626 & 1395 & 231 & 280 & 11200 \\
\hline & & & & & 337 & 4525 \\
\hline$\left[\mathrm{Ag}\left(6-\mathrm{Cl}-8-\mathrm{OCH}_{3} \mathrm{CA}\right)\right](\mathbf{3 g})$ & $1713 / 1682$ & 1617 & 1385 & 232 & 285 & 13400 \\
\hline$\left[\mathrm{Ag}\left(7,8-\mathrm{Cl}_{2} \mathrm{CA}\right)\right](\mathbf{3 h})$ & $1717 / 1693$ & 1619 & 1389 & 230 & 283 & 11025 \\
\hline \multirow[t]{2}{*}[\mathrm{Ag}(8-\mathrm{F}-6-\mathrm{BrCA})]{$(\mathbf{3 i})$} & 1693 & 1609 & 1398 & 211 & 275 & 9550 \\
\hline & & & & & $320^{c}$ & 3700 \\
\hline \multirow[t]{2}{*}[\mathrm{Ag}(6-\mathrm{OHCA})]{$(\mathbf{3} \mathbf{j})$} & 1713 & 1623 & 1416 & 207 & 280 & 10775 \\
\hline & & & & & 345 & 4800 \\
\hline$[\mathrm{Ag}(7-\mathrm{OHCA})](\mathbf{3 k})$ & 1706 & 1621 & 1384 & 237 & 325 & 15225 \\
\hline$[\mathrm{Ag}(8-\mathrm{OHCA})](3 \mathbf{l})$ & 1685 & 1595 & 1384 & 211 & 295 & 29825 \\
\hline \multirow[t]{2}{*}[\mathrm{Ag}(\text{phen})_{2}(6-\mathrm{ClCA})]{$\cdot 3.5 \mathrm{H}_{2} \mathrm{O}(\mathbf{4 d})$} & 1727 & 1620 & 1372 & 248 & 270 & 52550 \\
\hline & & & & & $328^{c}$ & 6275 \\
\hline \multirow[t]{2}{*}[\mathrm{Ag}(\text{phen})(8-\mathrm{Cl}-6-\mathrm{OCH}_{3}\mathrm{CA})]{$(\mathbf{4} \mathbf{f})$} & 1726 & 1617 & 1380 & 237 & 270 & 43025 \\
\hline & & & & & $342^{c}$ & 5525 \\
\hline$\left[\mathrm{Ag}(\text { phen })_{2}\left(6-\mathrm{Cl}-8-\mathrm{OCH}_{3} \mathrm{CA}\right)\right] \cdot 3.5 \mathrm{H}_{2} \mathrm{O}(\mathbf{4 g})$ & 1715 & 1630 & 1385 & 245 & 270 & 48500 \\
\hline
\end{tabular}

a All IR data was recorded as $\mathrm{KBr}$ discs.

b All UV-Vis data was recorded in DMSO.

c Shoulder.

Table 8

${ }^{1} \mathrm{H}$ NMR data for $\mathrm{Ag}(\mathrm{I})$ complexes $\mathbf{3 a}-\mathbf{l}$ and $\mathbf{4 d}, \mathbf{4}$ and $\mathbf{4 g}$, showing signal in ppm, multiplicity and $J$ values in $\mathrm{Hz}$ recorded in $\mathrm{d}_{6}$-DMSO.

\begin{tabular}{|c|c|c|c|c|c|}
\hline Compound & $\mathrm{C}-\mathrm{H}_{\text {vinyl }}$ & H5 & $\mathrm{H} 6$ & $\mathrm{H} 7$ & $\mathrm{H} 8$ \\
\hline$\left[\mathrm{Ag}\left(6-\mathrm{OCH}_{3} \mathrm{CA}\right)\right](\mathbf{3 a})$ & $6.37, \mathrm{~s}$ & $7.71, \mathrm{~d}, J=3.1$ & $3.77, \mathrm{~s}$ & $7.19, \mathrm{dd}, J=3.1,9.0$ & $7.30, \mathrm{~d}, J=9.0$ \\
\hline$\left[\mathrm{Ag}\left(7-\mathrm{OCH}_{3} \mathrm{CA}\right)\right](\mathbf{3 b})$ & $6.18, s$ & $8.04, \mathrm{~d}, J=8.8$ & $6.94, \mathrm{dd}, J=2.5,8.8$ & $3.86, \mathrm{~s}$ & $6.98, \mathrm{~d}, J=2.5$ \\
\hline$\left[\mathrm{Ag}\left(8-\mathrm{OCH}_{3} \mathrm{CA}\right)\right](3 \mathrm{c})$ & $6.35, \mathrm{~s}$ & 7.61, dd $J=2.4,7.0$ & $7.22, \mathrm{pt}, J=7.0,8.2$ & $7.26, \mathrm{dd}, J=2.4,8.2$ & $3.89, \mathrm{~s}$ \\
\hline$[\mathrm{Ag}(6-\mathrm{ClCA})](\mathbf{3 d})$ & $6.52, \mathrm{~s}$ & $8.33, \mathrm{~d} J=2.6$ & $\mathrm{Cl}$ & $7.67, \mathrm{dd}, J=2.5,8.8$ & $7.44, \mathrm{~d}, J=8.8$ \\
\hline$[\mathrm{Ag}(6-\mathrm{BrCA})](\mathbf{3 e})$ & $6.53, \mathrm{~s}$ & $8.45, \mathrm{~d}_{J}=2.5$ & $\mathrm{Br}$ & $7.76, \mathrm{dd}, J=2.4,8.8$ & $7.38, \mathrm{~d}, J=8.8$ \\
\hline$\left[\mathrm{Ag}\left(8-\mathrm{Cl}-6-\mathrm{OCH}_{3} \mathrm{CA}\right)\right](\mathbf{3 f})$ & $6.43, \mathrm{~s}$ & $7.73, \mathrm{~d}, J=2.9$ & $3.86, \mathrm{~s}$ & $7.42, \mathrm{~d}, J=2.9$ & $\mathrm{Cl}$ \\
\hline$\left[\mathrm{Ag}\left(6-\mathrm{Cl}-8-\mathrm{OCH}_{3} \mathrm{CA}\right)\right](\mathbf{3 g})$ & $6.49, \mathrm{~s}$ & $7.80, \mathrm{~d}, J=2.3$ & $\mathrm{Cl}$ & $7.36, \mathrm{~d}, J=2.3$ & $3.93, \mathrm{~s}$ \\
\hline$\left[\mathrm{Ag}\left(7,8-\mathrm{Cl}_{2} \mathrm{CA}\right)\right](\mathbf{3 h})$ & $6.52, \mathrm{~s}$ & $8.20, \mathrm{~d}, J=8.7$ & $7.62, \mathrm{~d}, J=8.7$ & $\mathrm{Cl}$ & $\mathrm{Cl}$ \\
\hline$[\mathrm{Ag}(8-\mathrm{F}-6-\mathrm{BrCA})](\mathbf{3 i})$ & $6.59, \mathrm{~s}$ & $8.31, \mathrm{~s}$ & $\mathrm{Br}$ & $7.91, \mathrm{dd}, J=2,10.0$ & $\mathrm{~F}$ \\
\hline$[\mathrm{Ag}(6-\mathrm{OHCA})](\mathbf{3 j})$ & $6.33, \mathrm{~s}$ & $7.52, \mathrm{~d}, J=2.9$ & $9.76(\mathrm{OH})$ & $7.01, \mathrm{dd}, J=2.9,8.9$ & $7.31, \mathrm{~d}, J=8 . \mathrm{S}$ \\
\hline$[\mathrm{Ag}(7-\mathrm{OHCA})](\mathbf{3 k})$ & $6.12, \mathrm{~s}$ & $7.93, \mathrm{~d}, J=8.8$ & $6.77, \mathrm{dd}, J=2.4,8.8$ & $10.60(\mathrm{OH})$ & $6.70, \mathrm{~d}, J=2.4$ \\
\hline$[\mathrm{Ag}(8-\mathrm{OHCA})](3 \mathbf{1})$ & $6.26, s$ & $7.46, \mathrm{dd}, J=1.7,8.0$ & $7.11, \mathrm{pt}, J=8.0$ & $7.07, \mathrm{dd}, J=1.7,8.0$ & $10.11(\mathrm{OH})$ \\
\hline$\left[\mathrm{Ag}(\text { phen })_{2}(6-\mathrm{ClCA})\right] \cdot 3.5 \mathrm{H}_{2} \mathrm{O}(\mathbf{4 d})$ & $6.62, \mathrm{~s}$ & $8.29, \mathrm{~d}, J=2.6$ & $\mathrm{Cl}$ & $7.63, \mathrm{dd}, J=2.6,8.9$ & $7.42, \mathrm{~d}, J=8.8$ \\
\hline$\left[\mathrm{Ag}(\right.$ phen $\left.)\left(8-\mathrm{Cl}-6-\mathrm{OCH}_{3} \mathrm{CA}\right)\right](\mathbf{4 f})$ & $6.46, s$ & $7.71, \mathrm{~d}, J=2.9$ & $3.80, \mathrm{~s}$ & $7.39, \mathrm{~d}, J=2.9$ & $\mathrm{Cl}$ \\
\hline$\left[\mathrm{Ag}(\text { phen })_{2}\left(6-\mathrm{Cl}-8-\mathrm{OCH}_{3} \mathrm{CA}\right)\right] \cdot 3.5 \mathrm{H}_{2} \mathrm{O}(\mathbf{4 g})$ & $6.35, \mathrm{~s}$ & $7.81, \mathrm{~d}, J=2.4$ & $\mathrm{Cl}$ & $7.23, \mathrm{~d}, J=2.4$ & $3.90, s$ \\
\hline
\end{tabular}

Table 9

${ }^{13} \mathrm{C}$ NMR data $(\mathrm{ppm})$ for the $\mathrm{Ag}(\mathrm{I})$ complexes, $\mathbf{3 a}-\mathbf{l}$ and $\mathbf{4 d}, \mathbf{4} \mathbf{f}$ and $\mathbf{4 g}$ recorded in $\mathrm{d}_{6}$-DMSO.

\begin{tabular}{|c|c|c|c|c|c|c|c|c|c|c|c|}
\hline Compound & $\mathrm{C} 2$ & $\mathrm{C} 3$ & $\mathrm{C} 4$ & $\mathrm{C} 5$ & C6 & $\mathrm{C} 7$ & $\mathrm{C} 8$ & C9 & $\mathrm{C} 10$ & $\mathrm{C} 11$ & $\mathrm{OCH}_{3}$ \\
\hline$\left[\mathrm{Ag}\left(6-\mathrm{OCH}_{3} \mathrm{CA}\right)\right](\mathbf{3 a})$ & 160.9 & 112.4 & 153.6 & 110.9 & 155.6 & 118.9 & 117.2 & 148.3 & 118.1 & 166.9 & 55.7 \\
\hline$\left[\mathrm{Ag}\left(7-\mathrm{OCH}_{3} \mathrm{CA}\right)\right](\mathbf{3 b})$ & 162.1 & 112.1 & 153.2 & 128.9 & 110.6 & 161.0 & 100.6 & 155.6 & 109.0 & 167.4 & 55.8 \\
\hline$\left[\mathrm{Ag}\left(8-\mathrm{OCH}_{3} \mathrm{CA}\right)\right](3 \mathrm{c})$ & 160.5 & 111.5 & 153.6 & 118.9 & 123.8 & 113.8 & 146.6 & 143.2 & 117.7 & 167.0 & 56 \\
\hline$[\mathrm{Ag}(6-\mathrm{ClCA})](\mathbf{3 d})$ & 160.3 & 114.1 & 151.6 & 127.2 & 128.0 & 131.3 & 118.5 & 152.4 & 118.8 & 166.7 & - \\
\hline$[\mathrm{Ag}(6-\mathrm{BrCA})](\mathbf{3 e})$ & 160.3 & 113.6 & 151.8 & 130.1 & 115.8 & 134.0 & 118.7 & 152.8 & 119.3 & 166.4 & - \\
\hline$\left[\mathrm{Ag}\left(8-\mathrm{Cl}-6-\mathrm{OCH}_{3} \mathrm{CA}\right)\right](\mathbf{3 f})$ & 159.9 & 113.2 & 153.5 & 110.5 & 154.8 & 118.5 & 120.2 & 143.6 & 119.2 & 166.5 & 55.9 \\
\hline$\left[\mathrm{Ag}\left(6-\mathrm{Cl}-8-\mathrm{OCH}_{3} \mathrm{CA}\right)\right](\mathbf{3 g})$ & 159.9 & 114.3 & 152 & 117.9 & 127.8 & 113.9 & 147.5 & 142.2 & 118.5 & 166.5 & 56.5 \\
\hline$\left[\mathrm{Ag}\left(7,8-\mathrm{Cl}_{2} \mathrm{CA}\right)\right](\mathbf{3 h})$ & 159.5 & 112.5 & 153.1 & 127.2 & 124.9 & 134.3 & 118.9 & 150.4 & 117.9 & 166 & - \\
\hline$[\mathrm{Ag}(8-\mathrm{F}-6-\mathrm{BrCA})](\mathbf{3 i})$ & 159.0 & 114.6 & 151.5 & $\begin{array}{c}124.7 \mathrm{~d} \\
J_{\mathrm{C}-\mathrm{F}}=3.5\end{array}$ & $\begin{array}{c}114.5 \mathrm{~d} \\
J_{C-F}=8.8\end{array}$ & $\begin{array}{l}120.3 \mathrm{~d} \\
J_{C-F}=20.5\end{array}$ & $\begin{array}{l}148.7 \mathrm{~d} \\
J_{\mathrm{C}-\mathrm{F}}=251\end{array}$ & $\begin{array}{l}141.4 \mathrm{~d}, \\
J_{\mathrm{C}-\mathrm{F}}=11.2\end{array}$ & 120.9 & 165.8 & - \\
\hline$[\mathrm{Ag}(6-\mathrm{OHCA})](\mathbf{3} \mathbf{j})$ & 161.0 & 111.8 & 153.7 & 112.3 & 153.4 & 119.7 & 117.1 & 147.1 & 117.8 & 167.4 & - \\
\hline$[\mathrm{Ag}(7-\mathrm{OHCA})](\mathbf{3 k})$ & 160.8 & 107.4 & 154.2 & 129.1 & 112.7 & 161.2 & 101.9 & 155.6 & 109.5 & 167.5 & - \\
\hline$[\mathrm{Ag}(8-\mathrm{OHCA})](3 \mathbf{1})$ & 160.8 & 110.6 & 155.2 & 117.7 & 123.7 & 117.9 & 142.6 & 144.6 & 118.3 & 167.2 & - \\
\hline$\left[\mathrm{Ag}(\text { phen })_{2}(6-\mathrm{ClCA})\right] \cdot 3.5 \mathrm{H}_{2} \mathrm{O}(\mathbf{4 d})$ & 160.0 & 115.6 & 149.0 & 126.9 & 128.0 & 131 & 118.3 & 152.4 & 118.4 & 165.0 & - \\
\hline$\left[\mathrm{Ag}(\right.$ phen $\left.)\left(8-\mathrm{Cl}-6-\mathrm{OCH}_{3} \mathrm{CA}\right)\right](\mathbf{4 f})$ & 159.8 & 114.2 & 151.6 & 110.6 & 154.9 & 118.5 & 120.2 & 143.7 & 119.1 & 165.1 & 56.0 \\
\hline$\left[\mathrm{Ag}(\text { phen })_{2}\left(6-\mathrm{Cl}-8-\mathrm{OCH}_{3} \mathrm{CA}\right)\right] \cdot 3.5 \mathrm{H}_{2} \mathrm{O}(\mathbf{4 g})$ & 160.3 & 112.2 & 154.3 & 119.3 & 127.5 & 113.6 & 142.3 & 147.4 & 118.8 & 166.3 & 56.5 \\
\hline
\end{tabular}


depending on the position of unsaturation. Also important to note is that $\alpha, \beta$-unsaturated and aryl conjugated acids show absorption for the carbonyl of the dimer at $1710-1680 \mathrm{~cm}^{-1}$ [33]. Therefore the $v_{\mathrm{C}=\mathrm{O}}$ band appearing at lower wavenumber in the IR spectra of the coumarin ligands may be assigned to $v_{\mathrm{C}=\mathrm{O}}$ (acid). Comparison of the spectra of the acids with those of the esters show that there is a new carbonyl band in the spectra of the acids which can be attributed to $v_{\mathrm{C}=\mathrm{O}}$ (acid).

The ${ }^{1} \mathrm{H}$ and ${ }^{13} \mathrm{C}$ NMR spectra of the ligands were recorded in $\mathrm{d}_{6^{-}}$ DMSO and the data are reported in Tables 4 and 5 respectively. The most characteristic signal in the ${ }^{1} \mathrm{H}$ NMR spectra of carboxylic acids is that of the $\mathrm{OH}$ group though not surprisingly in $\mathrm{d}_{6}$-DMSO it was not always observed ( $\delta_{\mathrm{OH}}$ varied from $14-9.82 \mathrm{ppm}$ ). A comparison of the ${ }^{1} \mathrm{H}$ NMR spectra of the acids with those of the corresponding esters shows the loss of the $-\mathrm{CH}_{3}$ of the methyl signal at approximately $3.9 \mathrm{ppm}$ confirming hydrolysis of the ester. The ${ }^{13} \mathrm{C}$ NMR signals of the coumarin acid ligands were assigned with the help of DEPT, HSQC and HMBC 2D experiments. The loss of the signal for the methyl ester carbon was observed in all of the spectra of the acids indicating that hydrolysis was complete.

\subsection{Synthesis and characterisation of the complexes}

The silver(I) complexes of the coumarin acids were formed by reaction with silver nitrate in aqueous solution in a $1: 1 \mathrm{M}$ ratio (Scheme 3). The acids were first deprotonated using $\mathrm{NaOH}$ prior to addition of silver nitrate. $\mathrm{All} \mathrm{Ag}(\mathrm{I})$ complexes were isolated as insoluble precipitates from the reaction media and when dried and stored in the dark they appeared to be air and moisture stable. The complexes were soluble in DMSO only. Elemental analyses of the complexes 3a-1, were in agreement with the proposed 1:1 ligand:silver ratio (Table 6). Only the phen-containing complexes of ligands $\mathbf{2 d}, \mathbf{2} \mathbf{f}$ and $\mathbf{2 g}$ were synthesised in acceptable purity. Previous studies have shown that inclusion of phen ligands in the coordination sphere of the cation not only improves solubility but has significantly improved bioactivity towards a number of clinically relevant species [34]. 4d, $\mathbf{4 f}$ and $\mathbf{4 g}$ were synthesised by firstly preparing the corresponding $\mathrm{Ag}(\mathrm{I})$ complex (3d, 3f and $\mathbf{3 g}$ ) and leaving the product in the reaction solution. A solution of 1,10-phen in ethanol was then added to this solution in a molar ratio of 1:1:2 ligand:metal:phen. The phen complexes were soluble in DMSO (hot) only. Elemental analyses indicated a metal to ligand ratio of $1: 1: 2$ for $\mathbf{4 d}$ and $\mathbf{4 g}$ with 3.5 water molecules in the formulation. A ratio of 1:1:1 ligand:metal:phen was observed for 4f (Table 6). All attempts at recrystallisation of the complexes isolated failed. Complex stability in DMSO solution was confirmed via measurement of ${ }^{1} \mathrm{H}$ NMR spectra of $\mathrm{d}_{6}$-DMSO samples of complexes stored over a period of months in the dark. No appreciable differences in the spectra were noted in this time.

\subsubsection{IR spectra}

The previously prepared complexes of coumarin-3-carboxylates allowed the identification of complexes, which whilst showing good antimicrobial activity, were polymeric in nature and had poor solubility in common organic solvents. We thought that might be related to the proximity of the carboxylate and lactone carbonyls which allowed for the formation of chelate complexes. Shifts of between 10 and $40 \mathrm{~cm}^{-1}$ in the lactone carbonyl seemed to confirm this coordination. Use of the 4-positon on the lactone ring for the carboxylate groups does not allow for the same type of coordination here and it was thought coordination to the carboxylate group alone would be favoured over bonding at the lactone carbonyl.

On examination of the IR spectra of the complexes, in comparison to those of the ligands, the most significant observation was the disappearance of the acid carbonyl band and the appearance of the asymmetric $\left(v_{\text {asym }}(\mathrm{OCO})\right)$ and symmetric $\left(v_{\text {sym }}\right.$ (OCO)) stretching frequencies. The $v_{\text {asym }}$ (OCO) and $v_{\text {sym }}$ (OCO) stretching frequencies and the $\Delta v(\mathrm{OCO})$ values for the $\mathrm{Ag}(\mathrm{I})$ complexes are given in Table 7 . The complexes (3a-1) had $\Delta v$ (OCO) values $>200 \mathrm{~cm}^{-1}$ indicating unidentate carboxylate coordination to the $\mathrm{Ag}(\mathrm{I})$ centre [35] and are consistent with those previously reported for $\mathrm{Ag}(\mathrm{I})$ complexes of coumarin-3-carboxylates [36]. Interestingly, the $v_{\mathrm{C}=\mathrm{O}}$ (lactone) stretching vibration was shifted in all of the complexes by $11-56 \mathrm{~cm}^{-1}$ relative to the free ligand, upon formation of the $\mathrm{Ag}(\mathrm{I})$ complexes as was observed in the coumarin-3-carboxylates. Several reports have attributed such a shift to the participation of the coumarin lactone carbonyl in complexation to the metal ion [37-39] but it has also been reported for other coumarin derivatives where the lactone was not involved in complexation $[40,41]$. In the latter instance the shift may be attributed to an increase in polarity of the lactone ring which can occur upon metal complexation. The insolubility of the complexes along with the IR data would suggest that they exist in the solid state as polymeric structures with extensive hydrogen bonding common to carboxylate structures as well as possible bonding of $\mathrm{Ag}(\mathrm{I})$ to both the deprotonated carboxylate oxygen and lactone carbonyl oxygen of

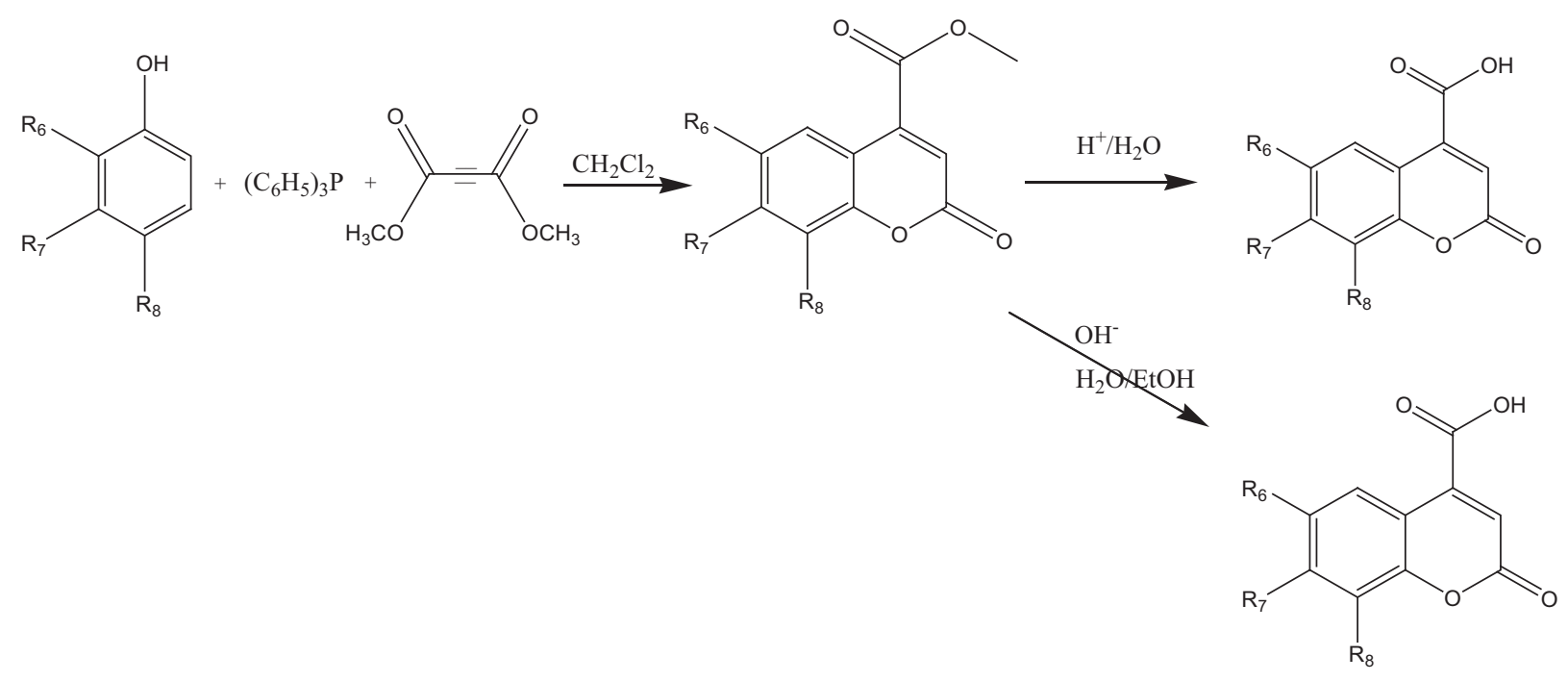


neighbouring ligands. Changes observed in the weak absorption bands at $400-600 \mathrm{~cm}^{-1}$ in the spectra of the complexes were assigned to the $\mathrm{M}-\mathrm{O}$ stretching vibrations $[42,43]$.

The IR spectra of the phen-containing complexes $\mathbf{4 d}, \mathbf{4 f}$ and $\mathbf{4 g}$ were similar to each other and showed new absorption bands characteristic of chelating phenanthroline [44], these occurring at ca. 1510,841 and $726 \mathrm{~cm}^{-1}$ (Table 7). The $\Delta v$ (OCO) values of the phen-complexes were also found to be $>200 \mathrm{~cm}^{-1}$ indicating unidentate coordination of the coumarin carboxylate group to the $\mathrm{Ag}(\mathrm{I})$ ion in all cases. The presence of water molecules was confirmed by the broad band observed at $\sim 3400 \mathrm{~cm}^{-1}$ in each of the spectra. The changes observed in the weak absorption bands at $400-600 \mathrm{~cm}^{-1}$ in the IR spectra of the complexes is attributed to the $\mathrm{M}-\mathrm{O}$ and $\mathrm{M}-\mathrm{N}$ stretching frequencies.

\subsubsection{NMR spectra}

Peak assignments (see Fig. 1 for atom numbering) for the ${ }^{1} \mathrm{H}$ and ${ }^{13} \mathrm{C}$ NMR spectra of all the $\mathrm{Ag}(\mathrm{I})$ complexes in $\mathrm{d}_{6}$-DMSO solution are given in Tables 8 and 9. In general, small shifts were observed for the aromatic protons upon complex formation but the greatest complexation induced shift (CIS) was observed for the vinyl proton H3 due to its proximity to the carboxylate group and this was shifted upfield by $0.45-0.56 \mathrm{ppm}$. The ${ }^{13} \mathrm{C}$ NMR spectra of the complexes revealed that the signals for the aromatic carbons (C5-C8) experienced a number of small shifts and the signal for the lactone carbonyl (C2) was mainly unaffected by complexation. The most significant shifts were observed for the carbons closest to the site of complexation, C3, C4 and C11. The signal for C4 experienced the greatest shift and was shifted downfield by 9.1-10.5 ppm. The carboxylate carbon, C11 was also shifted slightly downfield by 1-4 ppm and the vinyl carbon, C3 was found to be shifted slightly more upfield by 2-6.6 ppm in the spectra of the $\mathrm{Ag}(\mathrm{I}) \mathrm{com}$ plexes. Similar patterns were also reported for the spectra of the $\mathrm{Ag}(\mathrm{I})$ complexes of the coumarin-3-carboxylates [35].

From the NMR data of the complexes it is likely that binding of the coumarin ligands to $\mathrm{Ag}(\mathrm{I})$ in solution phase is through unidentate coordination of the carboxylate group. Comparison of the ${ }^{13} \mathrm{C}$ NMR spectra of the free ligands and their $\mathrm{Ag}(\mathrm{I})$ complexes (Tables 5 and 9) identified that the carbons of the carboxylate (C11) and lactone (C2) functionalities are only slightly deshielded upon complexation. This was also observed for the $\mathrm{Ag}(\mathrm{I})$ complexes of the coumarin-3-carboxylates and it was reported that the noted small shifts may be as a result of hydrogen-bonding present in the free ligands in DMSO solution.

The ${ }^{1} \mathrm{H}$ NMR spectra of the phenanthroline-containing complexes $\mathbf{4 d}$, 4f and $\mathbf{4 g}$ has four additional signals at ca. 9.17, 8.77, $8.21,8.01 \mathrm{ppm}$ corresponding to the protons of the phenanthroline ligands. The integrals confirm that the $2: 1$ phen:coumarin ratio for complexes $\mathbf{4 d}$ and $\mathbf{4 g}$ and the 1:1 phen:coumarin ratio for $\mathbf{4 f}$. On comparison with the spectra of the free phenanthroline ligand, small downfield shifts for the three of protons were observed in the spectra of all the complexes, similar to those noted for the mixed ligand $\mathrm{Ag}(\mathrm{I})$ complex of 4-hydroxy-3-nitro-2H-chromen-2one [25]. There are six signals observed in the ${ }^{13} \mathrm{C}$ NMR spectra of the complexes representing the non-equivalent carbons of the phenanthroline ligands all of which displayed relatively small CIS.

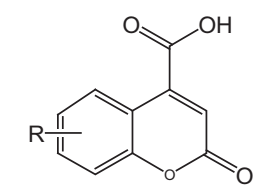

$\mathrm{R}=\mathrm{OCH}_{3}$

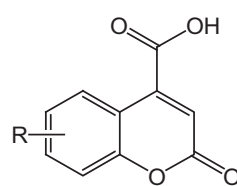

$\mathrm{R}=\mathrm{OH}$
Scheme 2. Demethylation of the methoxy substituted coumarin acids.

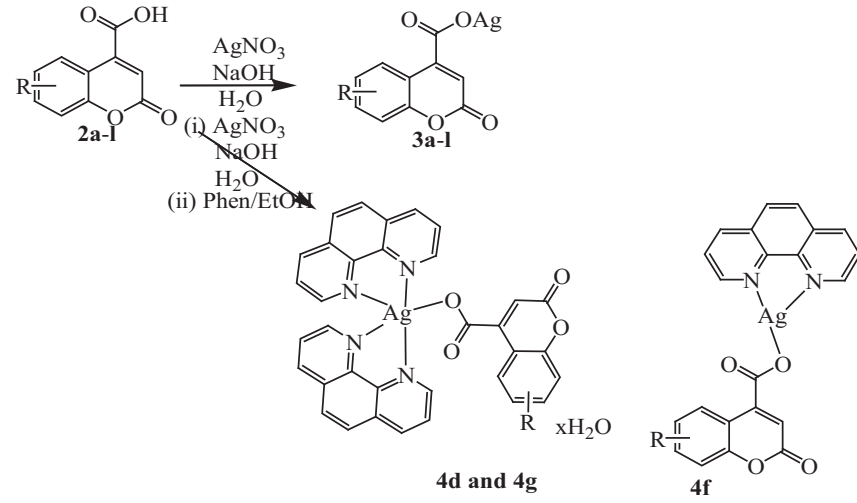

Scheme 3. General reaction scheme for synthesis of $\mathrm{Ag}(\mathrm{I})$ complexes of coumarin acids and phenanthroline adducts.

\subsubsection{Molar conductivity}

The molar conductivities of the $\mathrm{Ag}(\mathrm{I})$ complexes, 3a-1 (Table 6) were determined from conductance measurements obtained in DMSO solvent at $4.0 \times 10^{-3} \mathrm{M}$ concentrations. The values were in the range of $8.87-15.71 \mathrm{~S} \mathrm{~cm}^{2} \mathrm{~mol}^{-1}$ and were similar to those reported for other coumarin complexes recorded in DMSO and would suggest their non-electrolytic character [45]. The molar conductivity of the mixed ligand complexes $\mathbf{4 d}, \mathbf{4 f}$ and $\mathbf{4 g}$ was not recorded due to the poor solubility of the complexes in DMSO solvent.

\subsection{Antimicrobial activity of coumarin ligands and their $\mathrm{Ag}(\mathrm{I})$ complexes}

The $\operatorname{Ag}(\mathrm{I})$ complexes (3a-1, $\mathbf{4 d}, \mathbf{4 f}, \mathbf{4 g})$, the free ligands and the metal salt $\mathrm{AgNO}_{3}$ were screened for their activity against two Gram negative bacteria E. coli (NCIMB9485) and P. aeruginosa (ATCC 27853) and two strains of the Gram positive bacteria S. aureus, a methicillin sensitive strain, NCIMB12702 and a methicillin-resistant strain, ATCC4300 (MRSA). Commercial drugs vancomycin and gentamycin were also tested for their activities against relevant microbial strains. The antimicrobial activity of the compounds given in Table 10 and are expressed as $\mathrm{MIC}_{90}$ values (minimum concentration required to inhibit $90 \%$ of visible cell growth in $24 \mathrm{~h}$ ). Although a number of coumarin-derived compounds have previously shown antimicrobial activity [45], none of the synthesised metal-free coumarin ligands or phenanthroline showed any inhibition of growth at the maximum concentrations tested.

The $\mathrm{Ag}(\mathrm{I})$ complexes showed some antibacterial activity particularly against $E$. coli and $P$. aeruginosa. The activity of the complexes 3a-f, 3i-l tested against $E$. coli were slightly less than that of silver nitrate $\left(\mathrm{MIC}_{90}=36.1 \mu \mathrm{M}\right)$ and generally all were greater in activity than gentamycin $(60.3 \mu \mathrm{M})$. The type of substituent present on the benzene ring did not seem to influence their activity significantly against $E$. coli however the activity of the complexes (3a-h) tested against the P. aeruginosa ATCC 27853 did vary depending on the type of substituent present on the benzene ring. Complexes with a methoxy substituent only (3a-c) had similar activity and were comparable to that of $\mathrm{AgNO}_{3}(92.8 \mu \mathrm{M})$. The

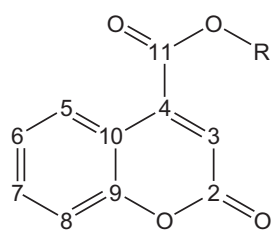

Fig. 1. Numbering system used to assign NMR spectra of coumarin-4-carboxylates and their $\mathrm{Ag}(\mathrm{I})$ complexes. 
Table 10

Antimicrobial Activity of Compounds ( $\mathrm{MIC}_{90}$ ).

\begin{tabular}{|c|c|c|c|c|}
\hline Compound & S. aureus NCIMB12702 & MRSA & E. coli NCIMB9485 & P. aeruginosa ATCC27853 \\
\hline$\left[\mathrm{Ag}\left(6-\mathrm{OCH}_{3} \mathrm{CA}\right)\right](\mathbf{3 a})$ & 123.1 & $>100$ & 75.3 & 94.9 \\
\hline$\left[\mathrm{Ag}\left(7-\mathrm{OCH}_{3} \mathrm{CA}\right)\right](\mathbf{3 b})$ & 174.7 & $>100$ & 46.6 & 94.0 \\
\hline$\left[\mathrm{Ag}\left(8-\mathrm{OCH}_{3} \mathrm{CA}\right)\right](3 \mathrm{c})$ & 188.0 & $>100$ & 47.3 & 94.2 \\
\hline$[\mathrm{Ag}(6-\mathrm{ClCA})](\mathbf{3 d})$ & 181.2 & $>100$ & 46.7 & 63.5 \\
\hline$[\mathrm{Ag}(6-\mathrm{BrCA})](3 \mathbf{e})$ & 102.6 & $>100$ & 47.1 & 75.3 \\
\hline$\left[\mathrm{Ag}\left(8-\mathrm{Cl}-6-\mathrm{OCH}_{3} \mathrm{CA}\right)\right](\mathbf{3 f})$ & 97.6 & $>100$ & 47.1 & 67.2 \\
\hline$\left[\mathrm{Ag}\left(6-\mathrm{Cl}-8-\mathrm{OCH}_{3} \mathrm{CA}\right)\right](\mathbf{3 g})$ & - & $>100$ & - & 52.8 \\
\hline$\left[\mathrm{Ag}\left(7,8-\mathrm{Cl}_{2} \mathrm{CA}\right)\right](\mathbf{3 h})$ & - & $>100$ & - & 90.5 \\
\hline$[\mathrm{Ag}(8-\mathrm{F}-6-\mathrm{BrCA})](\mathbf{3 i})$ & 139.5 & $>200$ & 46.9 & - \\
\hline$[\mathrm{Ag}(6-\mathrm{OHCA})](\mathbf{3 j})$ & 111.4 & $>200$ & 47.3 & - \\
\hline$[\mathrm{Ag}(7-\mathrm{OHCA})](\mathbf{3 k})$ & 108.2 & $>200$ & 46.9 & - \\
\hline$[\mathrm{Ag}(8-\mathrm{OHCA})](3 \mathbf{1})$ & 99.4 & $>200$ & 47.7 & - \\
\hline$\left[\mathrm{Ag}(\text { phen })_{2}(6 \mathrm{ClCA})\right] \cdot 3.5 \mathrm{H}_{2} \mathrm{O}(\mathbf{4 d})$ & - & $>100$ & - & 55.7 \\
\hline$\left[\mathrm{Ag}(\right.$ phen $\left.)\left(8-\mathrm{Cl}-6-\mathrm{OCH}_{3} \mathrm{CA}\right)\right](\mathbf{4 f})$ & - & $>100$ & - & 71.4 \\
\hline$\left[\mathrm{Ag}(\text { phen })_{2}\left(6-\mathrm{Cl}-8-\mathrm{OCH}_{3} \mathrm{CA}\right)\right] \cdot 3.5 \mathrm{H}_{2} \mathrm{O}(\mathbf{4 g})$ & - & $>100$ & - & 48.5 \\
\hline $\mathrm{AgNO}_{3}$ & 152. & $>100$ & 36.1 & 92.8 \\
\hline Ligands (2a-1) & $>200$ & $>200$ & $>200$ & $>100$ \\
\hline Phenanthroline & & $>100$ & & $>100$ \\
\hline Gentamycin & 33.5 & & 60.3 & \\
\hline Vancomycin & & 3.0 & & $>10$ \\
\hline Amphothericin B & & - & & - \\
\hline
\end{tabular}

complexes with halogen substituents (3d-h) were observed to have greater activity. Compounds with electron-withdrawing substituents such as halogens can have increased lipophilicity and hence an improved cell permeability of microorganisms. An increase in liposolubility is an important factor for antimicrobial activity and several groups have reported that the influence of a halogen substituent in a compound can affect its activity by steric or electronic effects [46-48]. The dichloro substituted complex $\left[\mathrm{Ag}\left(7,8-\mathrm{Cl}_{2} \mathrm{CA}\right)\right](\mathbf{3 h})$ showed activity $\left(\mathrm{MIC}_{90}=90 \mu \mathrm{M}\right)$ similar to the methoxy substituted complexes. The decrease in activity is likely as a result of its poor solubility and indeed meant that the complex had to be tested as a suspension.

Six of the complexes (3a-f) were also tested against $P$. aeruginosa (PA01) either in planktonic form or as a biofilm ( $\mathrm{MIC}_{90}$ values given in Table 11). Overall the activity of the substituted complexes against the planktonic strain was comparable to that found against $P$. aeruginosa ATCC 27853. The sensitivity of different strains of the same organism to a single antibiotic can depend upon the morphological and physiological characteristics of the strains [49] but there was no significant variation noted here.

The activity of silver and its complexes, including silver sulfadiazine, against $P$. aeruginosa biofilms has been studied previously by Bjarnsholt et al. and they found that the bactericidal concentration of silver needed to eliminate the bacterial biofilm was between 10 and 100 times greater than that used to eliminate planktonic bacteria [50]. Complexes $\mathbf{3 a}-\mathbf{f}$ and $\mathrm{AgNO}_{3}$ were found to be more active against the biofilm form of PA01 than the planktonic form and all BIC values were comparable. The complexes were approximately 4-6 times more potent against the biofilm than the planktonic form. This is clinically important as bacteria living in a biofilm can be up to 1000 times more resistant to antimicrobial agents than planktonic bacteria [51]. The antibacterial mechanism of silver ions has been well studied and it is possible that in these studies the silver complexes act by disrupting the signal processes associated with biofilm formation as the mechanisms involved in biofilm resistance to antimicrobials may differ from those responsible for antimicrobial resistance in planktonic bacteria. Biofilm resistance is multifactorial and includes a diffusion barrier, sequestration of antibiotics within the glucan polymers, slower growth, reduced oxygen concentrations at the base of the biofilm [52,53]. As such only a combination of different mechanisms could account for the levels of resistance seen in biofilm communities [54]. The fact that silver is said to have broad-spectrum activity in which it attacks multiple sites of microorganisms may be a factor in the
Table 11

Antimicrobial activity of selected $\mathrm{Ag}(\mathrm{I})$ complexes (3a-f) against Pseudomonas aeruginosa (PA01) expressed as $\mathrm{MIC}_{90}$ and $\mathrm{BIC}_{90}$.

\begin{tabular}{|c|c|c|}
\hline \multirow[t]{2}{*}{ Compound } & \multicolumn{2}{|c|}{ Pseudomonas aeruginosa (PA01) } \\
\hline & $\mathrm{MIC}_{90}(\mu \mathrm{M})$ & $\mathrm{BIC}_{90}(\mu \mathrm{M})$ \\
\hline Ligands (2a-f) & $>200$ & $>200$ \\
\hline$\left[\mathrm{Ag}\left(6-\mathrm{OCH}_{3} \mathrm{CA}\right)\right](3 \mathrm{a})$ & $95.8( \pm 0.4)$ & $25.0( \pm 1.3)$ \\
\hline$\left[\mathrm{Ag}\left(7-\mathrm{OCH}_{3} \mathrm{CA}\right)\right](\mathbf{3 b})$ & $96.6( \pm 1.4)$ & $18.3( \pm 3.6)$ \\
\hline$\left[\mathrm{Ag}\left(8-\mathrm{OCH}_{3} \mathrm{CA}\right)\right](3 \mathrm{c})$ & $93.2( \pm 0.4)$ & $19.6( \pm 3.8)$ \\
\hline$[\mathrm{Ag}(6-\mathrm{ClCA})](\mathbf{3 d})$ & $91.7( \pm 1.0)$ & $16.9( \pm 2.4)$ \\
\hline$[\mathrm{Ag}(6-\mathrm{BrCA})](3 \mathbf{e})$ & $154.2( \pm 30.2)$ & $21.2( \pm 0.5)$ \\
\hline$\left[\mathrm{Ag}\left(8-\mathrm{Cl}-6-\mathrm{OCH}_{3} \mathrm{CA}\right)\right](\mathbf{3 f})$ & $95.6( \pm 1.0)$ & $15.2( \pm 3.2)$ \\
\hline $\mathrm{AgNO}_{3}$ & $95.2( \pm 0.9)$ & $18.1( \pm 3.7)$ \\
\hline
\end{tabular}

$\mathrm{MIC}_{90}$ values (minimum concentration required to inhibit $90 \%$ of visible cell growth in $24 \mathrm{~h}$ ).

$\mathrm{BIC}_{90}$ values (biofilm inhibitory concentration, defined as the minimal concentration of compound to reduce biofilm formation by $90 \%$ after $18 \mathrm{~h}$ treatment.).

enhanced activity shown by the silver complexes against $P$. aeruginosa biofilm.

The phenanthroline containing complexes, $\mathbf{4 d}$, $\mathbf{4 f}$ and $\mathbf{4 g}$ and free phenanthroline were tested for their activity against MRSA (ATCC4300) and P. aeruginosa (ATCC 27853). Surprisingly their solubility in the solvent was poor and as such they were tested as suspensions. Metal-free phenanthroline has previously been reported to have significant activity against a number of microbes [55] however it was found to be inactive against the microbes used in this study $\left(\mathrm{MIC}_{90}>100 \mu \mathrm{M}\right)$. There is often variation in sensitivity of different strains of the same species to antimicrobial agents. Introduction of phenanthroline as a second ligand to the $\mathrm{Ag}(\mathrm{I}) \mathrm{com}-$ plexes improved the activity of $\mathbf{4 d}$ and $\mathbf{4 g}$ against $P$. aeruginosa only. The increase in activity would suggest a synergistic effect of both ligands and the silver ion. The $\mathrm{MIC}_{90}$ values for the compounds against MRSA were all greater than $100 \mu \mathrm{M}$ and so the introduction of phenanthroline did not increase the activity of the compounds against these microbes.

The activity of the complexes may be due to a synergistic effect of both ligand and metal ion or it may be as a result of the $\mathrm{Ag}(\mathrm{I})$ ion only. Silver has the ability to bind to the metal binding sites in proteins (sulfhydryl groups) thereby inactivating enzymes and blocking the respiratory chain of microorganisms [56]. The antimicrobial activity of several $\mathrm{Ag}(\mathrm{I})$ complexes of oxygen donor ligands is independent of whether the ligand itself possesses antimicrobial activity and results from the weaker bonding property of the $\mathrm{Ag}-\mathrm{O}$ bond 
and the ease of ligand replacement of the $\mathrm{Ag}(\mathrm{I})$ complexes with biological ligands such as those with $\mathrm{O}, \mathrm{N}$ or $\mathrm{S}$ donor atoms. The ligands are likely acting as carriers for the $\mathrm{Ag}(\mathrm{I})$ ion and the magnitude of the antimicrobial properties of the complexes is related to the ease with which they participate in ligand exchange reactions [57].

The complexation of the coumarin-4-carboxylates to $\operatorname{Ag}(\mathrm{I})$ resulted in enhanced antibacterial activity particularly towards $E$. coli and P. aeruginosa. Gram negative bacteria are generally less sensitive to biocides than Gram positive bacteria because of their outer membrane or envelope. The outer cell layer of Gram negative bacteria consists of an outer membrane, a thin layer of peptidoglycan and a periplasmic space and an inner membrane [58]. One of the effects of the outer envelope is to form an impermeable barrier that protects the microorganism from toxic molecules such as biocides and antibiotics. It allows the slow penetration of hydrophobic molecules and this decrease in penetration is responsible for the resistance of Gram negative bacteria to certain antibiotics. Several biocides can destabilise and disorganise the outer membrane thus gaining entry to the bacterial cell. Antibiotic passage may also occur by passive or facilitated diffusion through water-filled channels. The uptake and penetration process of the biocide will be influenced by interactions with all structures it encounters. Therefore the penetration of a biocide or antibiotic may often be determined by its physical properties rather than its specific structure. An optimal hydrophilic-lipophilic balance will enable the best penetration across the outer membrane and a complex mix of portioning and passive diffusion processes is thus likely to determine the progress of a biocide across the Gram-negative outer envelope [59]. It is therefore likely that the coordination of $\mathrm{Ag}(\mathrm{I})$ to the coumarin-4-carboxylate ligands results in a biocide with suitable physical properties which are capable of penetration of the outer membrane. The process in which it gains access is likely to be through an optimal hydrophilic-lipophilic balance. The increased activity of those complexes which had a halogen substituent also demonstrates the importance of lipophilicity for the activity of the complexes.

\section{Conclusion}

A number of novel $\mathrm{Ag}(\mathrm{I})$ coumarin-carboxylate complexes have exhibited moderate antimicrobial activity towards $E$. coli and $P$. aeruginosa. Some of the complexes showed greater activity against E. coli than gentamycin and were comparable in activity or greater than $\mathrm{AgNO}_{3}$ against $P$. aeruginosa grown planktonically or as a biofilm. Six of the complexes (3a-f) were approximately 4-6 times more potent against the biofilm compared to planktonic bacteria and introduction of phenanthroline as a second ligand to the $\mathrm{Ag}(\mathrm{I})$ complexes improved the activity of $\mathbf{4 d}$ and $\mathbf{4 g}$ against a strain of $P$. aeruginosa only. The poor solubility of the complexes would likely limit their use as potential oral drugs but they could be used in a number of therapeutic applications including coatings, medical devices, catheters, wound dressings, sutures etc. We have previously shown the potential of $\mathrm{Ag}(\mathrm{I})$ coumarin carboxylates for use as effective biocides in hygienic thin film coatings and the novel $\mathrm{Ag}(\mathrm{I})$ complexes in this study can now be investigated for use as additives for hygienic coatings.

\section{Acknowledgements}

This research was supported by the Centre of Applied Science for Health, ITT Dublin and by the Biology Department of National University of Ireland, Maynooth. The research was carried out by the Centre for Pharmaceutical Research and Development (CPRD) jointly located at Institutes of Technology, Tallaght and Dublin, and NUI, Maynooth, Co. Kildare, Ireland.

\section{Appendix A. Supplementary data}

Supplementary data associated with this article can be found, in the online version, at http://dx.doi.org/10.1016/j.poly.2013.09.042.

\section{References}

[1] D. Reed, S.A. Kemmerly, Ochsner J. 9 (2009) 27.

[2] E.M. Hetrick, M.H. Schoenfisch, Chem. Soc. Rev. 35 (2006) 780

[3] Trends in Staphylococcus aureus/MRSA bacteraemia in Ireland 1999-Q2 (2011)

[4] R. Kapoor, M.W. Wadman, M.T. Dohm, A.M. Czyzewski, A.M. Spormann, A.E. Barron, Antimicrob. Agents Chemother. 55 (2011) 3054

[5] T. Coenye, H.J. Nelis, J. Microbiol. Methods 83 (2010) 89.

[6] C.L. Fox Jr., Arch. Surg. 96 (1968) 184.

[7] I. Chopra, J. Antimicrob. Chem. 59 (2007) 587.

[8] P. Dibrov, J. Dzioba, K.K. Gosink, C. Hase, Antimicrob. Agents Chemother. 8 (2002) 2668.

[9] S.L. Percival, P.G. Bowler, D. Russell, J. Hosp. Infect. 60 (2005) 1.

[10] J.L. Clement, P.S. Jarrett, Met. Based Drugs 1 (1994) 5.

[11] Z. Zhao, K.A. Cornell, L.D. Hopman, B.L. Gibbins, SilvaSorb targeted antimicrobial activity. Medline. (<http://www.medline.com/wound-skincare/silvasorb/lit/Targeted.pdf>), 2003 (accessed 22.05.13).

[12] Patent US7135195, R.J. Holloday, H. Christensen, W.D. Moeller, (2006)

[13] M.A. Fonder, G.S. Lazarus, D.A. Cowan, B. Aronson-Cook, A.R. Kohli, A.J. Mamelak, J. Am. Acad. Dermatol. 58 (2008) 185.

[14] J. Markarian, Plastics, Additives and Compounding 6 (2004) 22.

[15] Plastics, Additives and Compounding 5 (2003) 32

[16] S. Jaiswal, K. Bhattacharya, M. Sullivan, M. Walsh, B. Creaven, F. Laffir, B. Duffy, P. McHale, Colloids Surf. B 102 (2013) 412.

[17] N. Hamdi, M.C. Puerta, P. Valerga, Eur. J. Med. Chem. 43 (2008) 2541.

[18] J.S. Lopez-Gonzalez, H. Prado-Garcia, D. Aguilar-Cazares, J.A. MolinaGuarneros, J. Morales-Fuentes, J.J. Mondoki, L. Cancer 43 (2004) 275.

[19] M.E. Riveiro, A. Moglioni, R. Vazquez, N. Gomez, G. Facorro, L. Piehl, E.R. de Celis, C. Shayo, C. Davio, Bioorg. Med. Chem. 16 (2008) 2665.

[20] J.R.S. Hoult, M. Paydt, Gen. Pharm. 27 (1996) 713.

[21] D.J. Hadijavlou-Litina, K.E. Litinas, C. Kontogiorgis, Med. Chem. 6 (2007) 293.

[22] R.H. Flatman, A. Eustaquio, S.M. Li, L. Heide, A. Maxwell, Antimicrob. Agents Chemother. 50 (2006) 1136.

[23] T. Lubbers, P. Angehrn, H. Gmunden, S. Herzig, Bioorg. Med. Chem. Lett. 17 (2007) 4708.

24] O. Kayser, H. Kolodziej, Z Naturforsch. 54 (1999) 169.

[25] B.S. Creaven, D.A. Egan, K. Kavanagh, M. McCann, M. Mahon, A. Noble, B. Thati, M. Walsh, Polyhedron 24 (2005) 949.

[26] E. Melliou, P. Magiatis, S. Mitaku, A.L. Skaltsounis, E. Chinou, L. Chinou, J. Nat. Prod. 68 (2005) 78

[27] S. Sardari, Y. Mori, K. Horita, R.G. Micetich, S. Nishibe, M. Daneshtalab, Bioorg. Med. Chem. 7 (1999) 1933.

[28] B.S. Creaven, M. Devereux, A. Foltyn, S. McClean, G. Rosair, V.R. Thangella, M. Walsh, Polyhedron 29 (2010) 813.

[29] S.M. Moskowitz et al., J. Clin. Microbiol. 42 (5) (2004) 1915.

[30] R. Hekmatshoar, Y.Sh. Beheshtiha, M.M. Heravi, K. Asadollah, Phosphorus Sulfur Silcon 177 (2002) 703.

[31] P.P. Kulkarni, A.J. Kadam, R.B. Mane, U.V. Desai, P.P. Wadgaonkar, J. Chem. Res. S. (1999) 394.

[32] B.S. Creaven, M. Devereux, I. Georgieva, D. Karcz, M. McCann, N. Trendafilova, M. Walsh, Spectrochimic. Acta, Part A 84 (2011) 275

[33] R.M. Silverstein, F.X. Webster, D.J. Kiemle, Spectrometric Identification of Organic Compounds, seventh ed., J. Wiley \& Sons, New York, 2005.

[34] B.S. Creaven, D.A. Egan, D. Karcz, K. Kavanagh, M. McCann, M. Mahon, A. Noble, B. Thati, M. Walsh, J. Inorg. Biochem. 101 (2007) 1108.

[35] G.B. Deacon, R.J. Phillips, Coord. Chem. Rev. 33 (1980) 227.

[36] B.S. Creaven, D.A. Egan, K. Kavanagh, M. McCann, M. Mahon, A. Noble, B. Thati, M. Walsh, Inorg. Chim. Acta 359 (2006) 3976.

[37] I.P. Kostova, I.I. Manolov, I.N. Nicolova, N.D. Danchev, Il Farmaco 56 (2001) 707.

[38] Tz. Mihaylov, N. Trendafilova, I. Kostova, I. Georgieva, G. Bauer, Chem. Phys. 327 (2006) 209.

[39] I. Kostova, G. Momekov, P. Stancheva, Metal-Based Drugs, 2007 (2007), Article ID 15925, 8pp.

[40] I. Georgieva, T. Mihaylov, G. Bauer, N. Trendafilova, Chem. Phys. 300 (2004) 119

[41] I. Georgieva, N. Trendafilova, B.S. Creaven, M. Walsh, A. Noble, M. McCann, Chem. Phys. 365 (2009) 69.

[42] J.R. Anacona, J. Gomez, J. Chil. Chem. Soc. 53 (2008) 1694.

[43] R.L. de Lima, L.R. de Souza Teixeira, T.M. Gomes Carneiro, H. Beraldo, J. Braz. Chem. Soc. 10 (1999) 184.

[44] J. Kaizer, T. Csay, G. Speier, M. Réglier, M. Giorgi, Inorg. Chem. Commun. 9 (2006) 1037.

[45] S. Khode, V. Maddi, P. Aragade, M. Palkar, P.K. Ronad, S. Mamledesai, A.H.M. Thippeswarmy, D. Satyanarayana, Eur. J. Med. Chem. 44 (2009) 1682. 
[46] D. Arish, M.S. Nair, Spectrochim. Acta, Part A 82 (2011) 191.

[47] V. Zelenák, K. Györyová, D. Mlynarcík, Met. Based Drugs 8 (2002) 269.

[48] S. Ravichandran, S. Sathish Kumar, Asian J. Biochem. Pharm. Res. 1 (2011) 136.

[49] S.A. Waksman, H.C. Reilly, A. Schatz, Bacteriology 31 (1945) 157.

[50] T. Bjarnsholt, K. Kirketerp-Møller, S. Kristiansen, R. Phipps, A.K. Nielsen, P.ø. Jensen, N. Høiby, M. Givskov, APMIS (2007) 921.

[51] T.B. Rasmussen, M. Givskov, Int. J. Med. Microbiol. 296 (2-3) (2006) 149.

[52] N. Hoiby, H.K. Johansen, C. Moser, Z. Song, A. Kjarazmi, Microbes Infect. 3 (2001) 23.
[53] T.-F. Mah, B. Pitts, B. Pellock, G.C. Walker, P.S. Steward, G.A. O’Toole, Nature 426 (2003) 306.

[54] E. Drenkard, Microbes Infect. 5 (2003) 1213.

[55] M. McCann, M. Geraghty, M. Devereux, D. O'Shea, J. Mason, L. O’Sullivan, Met. Based Drugs 7 (2000) 185.

[56] S. Franke, Microbiol. Monogr. 6 (2007) 343.

[57] S. Ahmad, A.A. Isab, S. Ali, A.R. Al-Arfaj, Polyhedron 25 (2006) 1633.

[58] J.-Y. Maillard, J. Appl. Microbiol. Sym. Suppl. 92 (2002) 16.

[59] S.P. Denyer, J.-Y. Maillard, J. Appl. Microbiol. Sym. Suppl. 92 (2002) 35. 